\title{
Lapurdum
}

Euskal ikerketen aldizkaria | Revue d'études basques |

Revista de estudios vascos | Basque studies review

$9 \mid 2004$

Numéro IX

\section{Place de Sainte Elisabeth de Portugal (1750) dans l'histoire des tragédies traditionnelles en langue basque}

\section{Bernard Oyharçabal}

\section{(2) OpenEdition}

Journals

Édition électronique

URL : http://journals.openedition.org/lapurdum/857

DOI : 10.4000/lapurdum.857

ISSN : 1965-0655

Éditeur

IKER

Édition imprimée

Date de publication : 1 novembre 2004

Pagination : 181-214

ISBN : 2-68781-376-X

ISSN : $1273-3830$

Référence électronique

Bernard Oyharçabal, « Place de Sainte Elisabeth de Portugal (1750) dans l'histoire des tragédies traditionnelles en langue basque », Lapurdum [En ligne], 9 | 2004, mis en ligne le 01 mars 2009, consulté le 19 avril 2019. URL : http://journals.openedition.org/lapurdum/857 ; DOI : 10.4000/ lapurdum. 857 


\title{
Place de Sainte Elisabeth de Portugal (1750) dans l'histoire des tragédies traditionnelles en langue basque
}

\author{
B. OYHARCABAL \\ IKER-UMR 5478
}

\section{$\S 1$. Introduction.}

Le but de cette recherche est de situer la tragédie traditionnelle ${ }^{1}$ Sa inte Elisabeth de Portugal (1750) dans l'histoire du théâtre tragique populaire basque, tel qu'il nous est connu à travers principalement son expression souletine. Après avoir indiqué quels sont les éléments caractérisant cette pièce à travers son manuscrit le plus ancien, sa thématique, sa composition, et son texte (\$2), je rappellerai, en m'appuyant sur la référence significative que représentent les mystères bretons, le contexte général dans lequel se situe la question de l'origine de cette tradition théâtrale (§3). En me fondant sur l'étude (Segurola 2002) des aspects matériels d'un manuscrit de Jeanne d'Arc, non daté mais considéré jusque là comme pouvant être plus ancien, je montrerai d'abord que la copie de Sainte Elisabeth du Musée Basque de Bayonne représente, en l'état actuel de nos connaissances, le manuscrit conservé le plus ancien relatif à cette tradition théâtrale spécifique $(\S 4)$, mais aussi comment il atteste du premier texte de tragédie traditionnelle connu. En effet, les pièces antérieures non conservées mais rapportées dans la littérature pour démontrer l'ancienneté des tragédies populaires basques (y compris hors de Soule), sont des supercheries littéraires, déjà dévoilées par Hérelle dans le cas de Marie de Navarre (\$5), ou non repérées jusqu'ici, pour la Sainte Geneviève labourdine, non datée, mais que Duvoisin assurait avoir oralement recueillie aux environs de 1830 (§6). Nous verrons, d'autre part, que les dates d'ex-libris plus anciennes que celle des copies, comme celle de 1634 qui apparaît dans un manuscrit de Sa int Jacques du XIXe siècle (Hérelle 1926b, Urkizu 1990), résultent en réalité d'une mauvaise lecture, sans qu'il n'y ait, selon nous, aucun doute à ce sujet (§7). C'est la même analyse qui doit être préférentiellement retenue, mais sur

1 - Le terme de tragédie désigne ici les pastorales à sujets tragiques dans l'appellation de Hérelle (1926b), telles qu'elles furent représentées en Soule jusqu'à la première guerre mondiale de manière traditionnelle. La désignation de pastorale est généralement utilisée aujourd'hui, mais dans la mesure où ce dernier vocable désigne également, d'une part, les formes modernisées de ce théâtre, et d'autre part, un genre dramatique caractérisé par ses personnages et sa thématique, par commodité, nous utilisons de préférence celui de tragédie (traditionnelle) pour nous référer au répertoire et aux modes de représentation antérieurs à 1914 du théâtre populaire. 
le mode hypothétique cette fois, compte tenu de l'absence de document, au sujet du manuscrit de Clovis que Buchon (1839), le premier éditeur des Chroniques Nationales françaises des XVe et XVTe siècles, datait, de manière sans doute bien légère, de l'an 1500 (§8). Dans une dernière partie, reprenant les termes d'une comparaison avec les attestations d'un théâtre basque du XVIe siècle, j'indiquerai en premier lieu en quoi les diverses affirmations concernant la présence d'acteurs navarrais à la fin du XVle (Floristán 1993, Satrústegi 1993, Urkizu 1996) fondées sur la correspondance navarro-castillane de Charles de Luxe (1595) sont le résultat d'une leçon très improbable des textes ( $\$ 9$ ). Je montrerai, en second lieu, pourquoi, au vu de témoignages indirects mais fiables tels que celui d'Oihenart (1665), la tradition théâtrale populaire souletine ne paraît pas constituer un prolongement des pastorales bas-navarraises du XVIe siècle, mais relève probablement d'une source indépendante plus tardive $(\S 10)$. Je conclurai par quelques considérations plus générales concernant la littérature écrite produite hors du contexte de l'impression. En effet, si les tragédies traditionnelles souletines représentent dans le passé l'exemple majeur de ce secteur de la production littéraire en langue basque, il est probable que c'est un domaine qui a dû dans les siècles précédents, notamment au XVIe siècle, être plus riche, même si fort peu de documents ont été conservés de ces écrits éphémères (§11).

Cette recherche, à côté de son aspect purement informatif et historique, a également pour objectif de montrer à travers un cas pratique divers pièges qui peuvent apparaître en matière de datation de tradition littéraire populaire, genre pour lequel on peut s'attendre à un certain décalage entre la date supposée de sa naissance et celle des premières attestations documentées le concernant. Pour cette raison, je reprends ici un certain nombre des arguments déjà utilisés dans un article précédent rédigé en basque sur cette question (Oyharçabal 1999), car la question de la datation du théâtre tragique traditionnel souletin est fort instructive, tant elle a donné lieu depuis le XIXe siècle à des supputations hasardeuses.

\section{\$2. La tragédie de Sainte Elisabeth de Portugal.}

§2.1. Le manuscrit de Sainte Elisabeth de Portugal, point de départ de notre recherche (mss. 14 du Musée basque, Fonds Campan-Latsague déposé en 1926) et dont nous préparons une première édition, est décrit par Hérelle (1928) dans son catalogue du répertoire du théâtre tragique souletin de la manière suivante $:^{2}$

Musée basque, $n^{\circ} 14$. Cahier couvert en papier gris ; papier vergé, 310 sur $210 \mathrm{~mm}$. ; 74 pages à 2 colonnes. Complet; 1596 versets comptés par le copiste. Ex-libris da té : Ga uliber, 1750. XVIII siècle (Le répertoire du théâtre tragique, Ca talogue a nalytique, p. 88).

Les indications de Hérelle sont exactes dans l'ensemble, les seules corrections à apporter portant sur le nom du copiste auteur de l'ex-libris, qui est plutôt, selon ma lecture, Jauliber, et sur le nombre de pages: le nombre de pages comptées par le copiste est en réalité de 72 , Hérelle ayant rajouté les deux pages de la dernière feuille (contenant l'épilogue), et qui ne sont pas comptées dans le manuscrit. Mais, en réalité, il y a en tout 39 feuilles, écrites recto verso, soit 78 pages, dont une

2 - Il ne s'agit pas d'ailleurs du manuscrit unique. Hérelle (1928, p. 88) mentionne également le manuscrit 208 de la Bibliothèque Nationale, et un fragment sur le manuscrit des Douze Pairs de France, dans la Bibliothèque Larrieu. 
toutefois ne contient pas de versets. Il s'agit du verso de la feuille 2 (4e page), ${ }^{3}$ laquelle vient entre le prologue et le texte de la tragédie proprement dite, cette dernière débutant au recto de la feuille 3 . Le décalage dans le dénombrement des pages provient de ce que les pages du prologue (pages 1 à 3) sont comptées à part. La pagination du manuscrit est donc la suivante : 3 feuilles avec pages numérotées par le copiste de 1 à 3 (prologue) et une page non comptabilisée (sans texte se rapportant à la tragédie) ; puis, 36 feuilles avec pages numérotées par le copiste de 1 à 72 , contenant la totalité de la tragédie sauf les vingt-deux derniers versets ; enfin, une feuille sans numéro de pages, contenant, au recto, les vingt-deux derniers versets de la tragédie, et au verso, les versets de l'épilogue, l'ex-libris, la liste des personnages avec le nombre de versets à dire par chacun d'eux, l'ordre par rang pour l'entrée en scène, une liste de dates.

La question se pose de déterminer si l'ensemble du manuscrit est de la même main. Tel me paraît être le cas pour les 38 premières feuilles, mais un doute très fort surgit quant à la dernière (rappelons qu'elle est la seule à ne pas être numérotée dans la pagination), car la graphie, bien que proche, est dissemblable, et les écarts nets sur certains points d'orthographe (avec, par exemple, l'usage de $y$ en fin de mot, qui n'apparaît pas dans les feuilles précédentes). Dans l'hypothèse, très probable, d'une seconde main, le copiste de ce manuscrit de tragédie ne serait donc pas, pour l'essentiel, Jauliber, dont l'ex-libris apparaît sur cette dernière feuille. Reste, cependant, que ce dernier en était le propriétaire au moment de la représentation d'Esquiule de 1750.

Comme l'indique Hérelle, le manuscrit est complet, incluant notamment le prologue (62 versets) et l'épilogue (12 versets). L'ex-libris, que nous reproduisons dans la figure 1, est de la main du copiste de la dernière feuille et comprend une indication sur la date, le lieu de la représentation et le nom du régent également propriétaire de la copie :

Fa it à Ezkioula le trois janvier 1750

Ceux et celle qui trouveron le presant

Seront prié deremettre a moy à ja uliber

De la ditte commune il leur sera recompence

$<>$ leur < peine?

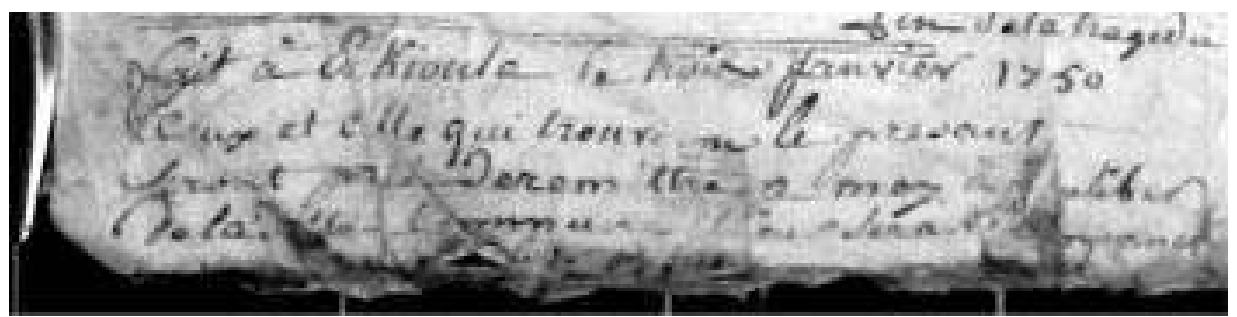

Fig. 1. Ex-libris du manuscrit de Sa inte Elisabeth de Portugal (1750)

Cet ex-libris est précédé d'une indication sur le titre de la tragédie, dont la seconde partie est écrite verticalement en milieu de page et suivie du comptage des versets :

3 - Cette page n'est pas vierge. Y figurent diverses indications d'ordre domestique, dont certaines datent du XIXe siècle, et qui sont sans rapport avec la tragédie. 
Fin de la tragedie de Ste Elisabeth / ylia Entout 1596 vers

Outre le texte de la tragédie, le manuscrit comprend sur sa dernière page :

- une liste des rôles avec le nombre de versets correspondant à chacun de ces rôles sous le titre noms des acteurs. 44 rôles sont mentionnés par ordre de première apparition.

- une série de listes de composition des rangs par rôles : 5 rangs sont mentionnés comprenant chacun une liste de 4 à 6 noms de personnages (au total, 25 personnages). Ces rangs doivent correspondre à l'ordre d'apparition des acteurs sur scène en début et en fin de représentation. ${ }^{4}$

- une liste de 13 dates, avec mention du saint du jour, en deux séries séparées par une double ligne de 10 et 3 dates respectivement, la seconde correspondant à des évangélistes :

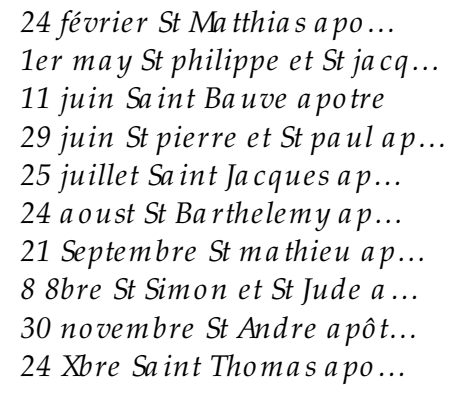

24 avril St marc evangeliste

18 8bre St Luc evangeliste

$27 \mathrm{Xbre}$ St Jea $n$ evangeliste

Cette liste est écrite de la même main que le reste de cette feuille du manuscrit, et matériellement elle vient à la fin du document, à la suite des listes des rangs. Quelle est sa signification ? Je l'ignore. Apparemment, sa disposition en fin de manuscrit autorise à la mettre en rapport avec la tragédie, mais de quelle manière ? Peut-on penser, par exemple, qu'il s'agit de dates pour lesquelles, en dehors de la représentation du 3 janvier, d'autres avaient été également envisagées? Ceci est peu probable, car ordinairement jusqu'à la fin du XIXe siècle, époque à laquelle on prit l'habitude de jouer les tragédies à deux, et plus rarement à trois reprises, les représentations n'avaient lieu qu'une seule fois (Hérelle 1922, p. 225). ${ }^{5}$ Peut-être le fait que les saints évangélistes soient listés à part indique-t-il qu'il s'agissait de listes ayant une signification particulière pour le copiste, mais sans rapport avec la tragédie elle-même?

§2.2. La tragédie Sainte Elisabeth est représentative dans sa thématique des pastorales traditionnelles qui représentaient la vie d'un personnage principal dit süjet, en l'occurrence une sainte, également personnage historique, puisque reine. Rien dans le manuscrit ne donne à penser qu'il s'agissait d'une représentation qui

4 - Comme on peut le constater, le nombre d'acteurs figurant sur les listes des rangs est nettement inférieur à celui des personnages. Ceci s'explique par le fait qu'un même acteur pouvait jouer plusieurs personnages.

5 - On peut se demander, toutefois, si certaines tragédies n'ont pas été jouées dans des lieux différents et par l'entremise de régents différents la même année, après cession de copie. C'est ce que donnent à croire les deux ex-libris figurant sur la copie d'CEdipe (Bilbao 1996), l'un de Bohoteguy (mars 1793), et le second de Mecol (juin 1793). 
était destinée à être jouée par des femmes, bien que cette possibilité existât pour des tragédies à süjet féminin. Au demeurant, Sainte Elisabeth ne fait pas partie des quelques pastorales répertoriées comme ayant été jouées par des actrices (Sa inte Hélène de Constantinople, Sa inte Geneviève de Brabant, Sa inte Marguerite, et Sa inte Engrâce ; cf. Hérelle 1922, p. 337).

Le thème de la tragédie peut être caractérisé comme relativement moderne : en effet, il est basé sur la vie de la fille du roi d'Aragon, née en 1271, qui épousa Denis 1 er roi de Portugal, et mourut en 1336, quelques années après ce dernier. La dévotion à son égard ayant été promue par la monarchie portugaise, elle fut canonisée en 1625, et on peut imaginer que c'est en adaptant la littérature hagiographique née de cette canonisation que la tragédie fut rédigée en basque, sans que l'on soit en mesure de définir une date pour cette adaptation.

§2.3. Les divers épisodes représentés dans la tragédie appartiennent pour l'essentiel à l'histoire de la sainte, avec certaines scènes comiques ajoutées en marge du récit représenté. On peut résumer la tragédie, en six grands épisodes, de la manière suivante :

- préparation du mariage imposé par son père à la jeune Elisabeth, âgée de 12 ans et très dévote, avec le roi de Portugal ;

- infortunes de la vie d'une épouse modèle de charité et de fidélité avec un mari débauché, comprenant notamment un épisode au cours duquel, un proche d'Elisabeth, don Pedro, est faussement accusé d'être l'amant de la reine par un courtisan; ce dernier voudrait que le roi, se croyant trompé, répudie la reine pour épouser sa sœur ; mais, don Pedro, qui s'est opportunément arrêté dans une église, est sauvé de la mort, à laquelle il est voué du fait d'un stratagème du roi jaloux ; le courtisan calomniateur meurt à sa place, jeté dans un four à chaux (épisode précédé d'une scène bachique entre les chaufourniers) ; le roi voit en cela un signe divin et se rend compte de sa méprise et de ses erreurs.

- Alfonsa, fils héritier du trône, se révolte contre son père, ce qui entraîne des guerres entre père et fils; au cours de ce conflit, le roi Denis, qui accuse à tort son épouse d'être complice de son fils, éloigne Elisabeth en exil ; mais la lecture d'une lettre permet d'innocenter la reine, tout ceci conduisant à la fin des guerres et à une réconciliation générale.

- maladie et mort édifiante de Denis, veillé par son épouse, ceci donnant lieu à un jeu classique dans ce théâtre sur le thème de la médecine ; Elisabeth entre dans un couvent qu'elle a fait bâtir, ce qui offre le prétexte à une scène de disputes entre deux artisans ; Elisabeth se rend ensuite à Compostelle en pèlerinage.

- épisode guerrier, entre Alfonsa, devenu roi de Portugal et Christophle, roi de Grenade, son fils (et donc petit-fils d'Elisabeth) ; ils se réconcilient grâce à l'action pacificatrice d'Elisabeth, qui meurt saintement après que la vierge lui fut apparue, et qu'elle eut recommandé de combattre les Maures.

- nouvelle guerre d'Alfonsa et Christophle contre Magot, roi du Maroc; ce dernier est vaincu et tué malgré l'aide de Rodrigas (forme généralement utilisée dans le texte à côté de Rodrigues préféré dans les didascalies), prince de Grenade, et de Pascalina, l'ancienne maîtresse de Denis; ces deux derniers meurent également ; le premier, fait prisonnier, est brûlé, et la seconde, qui est parvenue à s'enfuir, frappée par la foudre. Il est fort possible que ce dernier épisode, où Elisabeth personnellement n'intervient plus, résulte d'une contamination d'une autre tragédie. 
§2.4. Le texte de Sa inte Elisabeth se présente en quatrains séparés par une ligne, écrits sur deux colonnes par pages. Conformément à la tradition ancienne, ces vers n'ont pas de mesure et ne satisfont de façon régulière qu'à une règle d'assonance sur les deux vers pairs de chacun des quatrains. Les rimes riches résultent généralement, non d'un effort particulier, mais de la reprise en parallèle de suffixes fonctionnels identiques, comme, par exemple, les participes futurs (abandonatüren ... kondenatüren) ou les formes locatives des phrases nominalisées (erhaitera ... akisitzera).

Toujours dialogué, le texte abonde en formes impératives et modales, en se conformant au rythme imposé par le mode de déclamation : le verset est l'unité maximale contenant un énoncé complet, et le plus souvent il en contient deux, correspondant aux deux vers assonancés : les coupures d'hémistiche, qui sont marquées dans la graphie (comme dans le mode déclamatoire) correspondent à une coupure syntagmatique, les contre-exemples étant suffisamment rares pour être considérés comme des exceptions.

Aucun élément du vocabulaire ou de la syntaxe ne signale un décalage par rapport à la datation du manuscrit. L'orthographe est celle que l'on rencontre généralement dans les manuscrits de tragédies souletines, étant entendu qu'il ne s'agit en aucune façon d'un système orthographique standardisé. Par exemple, les phénomènes de fermeture des voyelles médianes emaztiari [emazte+ ari], ukheitia [ükheite+a], hitcian [hitz+ean], gincoua [jinko+a] khorouari [khorua+ari], palaciouan [palazio + an], ...), tout comme le désarrondissement de $\ddot{u}$ (celia [zelü+ a], falxia [faltsü+ a], dian [dü+ an], ...) sont invariablement transcrits. De même le contraste entre $u$ et $\ddot{u}$ est systématiquement restitué par l'emploi de $o u$ vs $u$ (coure vs çuc).

En ce qui concerne les consonnes, la principale graphie particulière consiste à employer des finales en $-x t$ dans des vocables se terminant par l'affriquée apicale (sinext, erakaxt,...), alors qu'à l'intérieur des mots cette affriquée est transcrite $x$ à l'intervocalique (ourtouxik, laxun) ou après consonne (penxatzen, ounxa, ...) ; ce digramme apparaît aussi avec l'affriquée palatale (hanixt). Pour le reste, nous signalerons uniquement l'emploi fréquent de la lettre $k$, car on a pu voir en ceci un indice de rédaction tardive de la copie. Faut-il donc considérer cet usage comme un signe de postériorité du manuscrit par rapport à la date de l'ex-libris, au motif que l'emploi régulier de $k$ pour rendre l'occlusive vélaire sourde ne se serait imposé que tardivement dans l'orthographe souletine $?^{6}$ Non, car l'emploi non généralisé, mais régulier, de $k$ fut relativement précoce (antérieur au XIXe siècle) dans divers textes souletins, de sorte que l'usage de cette lettre ne représente en aucune façon une innovation qui signalerait nécessairement ici un décalage entre la copie et l'ex-libris. Dans le cas présent, le catéchisme de Belapeyre (1696), auteur du premier ouvrage proprement souletin publié, constitue une bonne référence. Ainsi que le montre Agirre (1996, p. 98-106), en dehors de son emploi pour restituer l'aspirée $(k h)$, la lettre $k$ est déjà présente chez Belapeyre, de façon régulière devant $e$ et $i$, et de façon lacunaire devant les autres voyelles. A l'inverse, elle est exclue en fin de mot et devant consonne. Dans la copie du Musée Basque de Sa inte Elisabeth, les choses

6 - On peut voir une manifestation de l'incertitude qui demeura longtemps sur ce point en souletin dans les remarques que Saroïhandy fit paraître en appendice à son édition de la pastorale de Roland (1927, p. 126-7). Il y explique sa préférence en faveur de $q$ plutôt que de $k$ (alors que le catéchisme souletin de 1913 avait adopté cette dernière lettre), faisant ainsi écho à une question orthographique clairement exprimée par les choix opposés faits implicitement, près de deux siècles plus tôt, par les copistes des deux premiers manuscrits de tragédies connus (voir note suivante). 
se présentent globalement de manière parallèle : l'emploi de $k$ est régulier avec l'aspirée ( $k$ : cherkhaturen, jalkhi, ikhousten ...), et exclu en fin de mot (au profit exclusif de $c$ : nic, neska tilec, ederric, erreguec, ja unac, jhourc...). Devant voyelle, $k$ est également employé devant $e$ (gunuke, banikeçu, ...) et $i$ (adiskide, hareki, ...). La seule différence entre les deux graphies porte sur le fait que le copiste de Sa inte Elisabeth, contrairement à Belapeyre, étend l'usage préférentiel de $k$, pour l'utiliser devant a (neskatilac, esplikatcen,...) et o (kokia, fra nko, hartako, malgré l'emploi systématique de $c$ dans ginco et ses dérivés) et, de manière moins régulière, devant $u$ (ezkount et ezcount). Il semblerait, au demeurant, que cet usage étendu de $k$ ne soit pas sans précédent selon ce que rapporte dans sa thèse sur l'histoire de l'orthographe des textes basques septentrionaux des XVIe et XVIIe siècles Mujika (1997, p. 583). Il observe, en effet, à propos du catéchisme souletin de 1706 (que je n'ai pu quant à moi consulter) : herska ri bela rea a dierazteko k erabiltzen du honek ere eta ez e eta i bokalen a urrean bakarrik (lui aussi [le traducteur en basque de ce catéchisme] utilise également $k$ pour l'occlusive vélaire, et pas seulement devant les voyelles e et $i$ ).

Cette tendance à la régularisation de l'orthographe du [k] (ici limitée au positionnement prévocalique) n'est en rien anachronique par rapport à la date de 1750 dans le contexte des copies de tragédies souletines. On peut le constater en comparant la graphie de [k] dans cette copie de Sainte Elisabeth avec celle employée dans le second manuscrit de tragédie connu (Jean de Paris, 1760), où ladite tendance est également très présente, mais cette fois-ci, au bénéfice de la lettre $q .^{7}$ On voit par là que l'orthographe du copiste de Sainte Elisabeth est beaucoup plus proche du modèle premier de Belapeyre (et du catéchisme d'Oloron traduit en souletin en 1706) que celle d'Arhex, copiste de Jean de Paris, manuscrit postérieur d'une dizaine d'années seulement.

\section{§3. La tradition des tragédies souletines, expression du théâtre populaire régional européen.}

Les tragédies souletines représentent une expression du théâtre populaire que connurent diverses régions d'Europe jusqu'au XIXe siècle. ${ }^{8}$ En France, ce théâtre fut présent au-delà du XVIe siècle, notamment, mais pas uniquement, dans les campagnes des pays linguistiquement non romans comme la Bretagne, les Flandres ou le Pays basque. Comme il s'agissait d'un théâtre fait uniquement par et pour les villageois, il s'exprimait dans les langues de ces régions, et par conséquent, en Soule, province orientale du Pays basque, les tragédies traditionnelles étaient d'expression basque, et plus précisément souletine.

7 - Dans ce manuscrit de Jean de Paris (mss 19 du Musée Basque), en effet, la lettre $q$ (suivie de $u$ ) est employée devant $e$ et $i$, ce qui n'est pas étonnant compte tenu du modèle français (eztaquit, adisquidia, nuquecu, cuntuquet,...), mais elle est aussi systématiquement utilisée en fin de mot (jaunaq, niq, ihourq, besteriq, ...), la lettre $c$ (comme bien sûr $k$ ) étant exclue dans cette position. En dehors des orthographes étymologiques dans les emprunts, du type comissionia, ocupatia, countent, coraja, ...), le copiste utilise aussi fréquemment $q u$ devant $o$ (hanquo, cer gradotaquo guiçon, beçalaquo, erostequo, malgré, ici aussi, ginco) et même devant a (besarqua, cherqua, benediqua, eraqatx / eraquastia, pharqua, ... à côté de ca mbarala , pharca, benedicatia,...). Le $q$ apparaît également devant $\ddot{u}$ (esqutiq) et $u$ (qoulierbat, à côté de ezcouncia). Avec l'aspirée également $q$ remplace régulièrement le $k$ rencontré dans Sa inte Elisabeth : iqhousi, uqhen, qhumitu,...

8 - Ce théâtre est peu connu dans son contenu. Les pièces publiées à ce jour n'atteignent pas la dizaine, la plupart sans traduction: St Julien d'Antioche (Vinson, 1891), Hélène de Constantinople (Léon, 1909), de façon non suivie, mais avec traduction en français), Roland (Saroïhandy, 1927, publication partielle, avec traduction en français), Cha rlemagne (Oyharçabal 1981, avec traduction en français), Jean de Pa ris (Mozos 1994), Sa int Ja cques (Urkizu 1990). CEdipe (Bilbao, 1996) et Sa inte Ca therine (Loidi, 1996). 
Ce théâtre populaire ayant conservé certains traits caractéristiques des représentations dramatiques médiévales, il ne fait pas de doute qu'un lien de filiation doit être établi avec celles-ci, ce que les spécialistes ont fait depuis le XIXe siècle. Il serait déraisonnable, en effet, de supposer que les Souletins eurent réinventé sans modèle les éléments structurants du théâtre populaire issu de la tradition dramatique médiévale. De la même manière, seule une source commune directement ou indirectement dérivée de celle-ci, évidemment plus tardive, peut expliquer les remarquables similitudes qui caractérisent les dispositifs scéniques des mystères bretons et des tragédies souletines. Les coïncidences observées entre les unes et les autres ne sauraient résulter du hasard, ni, bien sûr, de quelque archétype naturel ou primitif des représentations dramatiques. Il suffit de considérer les descriptions de la scène des mystères bretons qui nous ont été laissées par les auteurs du XIXe siècle pour constater leur ressemblance avec celle des tragédies souletines. Voici par exemple la description de la scène des représentations bretonnes du XIXe siècle offerte par Le Braz (1905), qui suit Luzel ${ }^{9}$ sur ce point :

Une estrade en bois élevée d'un peu plus d'un mètre au-dessus du sol, et dont les planches sont plus ou moins bien ajustées, reposaient soit sur des barriques, soit sur des chevalets. Sa longueur éta it d'environ quinze pas sur huit de profondeur. Vers le tiers de la largeur étaient accrochées à des montants verticaux deux vastes pièces de toile qui partaient obliquement de chaque côté de l'estrade et se rejoignaient en son milieu de façon à former un angle rentrant.

Cette estrade est construite très simplement. Sur une triple rangée de barriques mises debout et reliées par des solives, on cloue des planches épaisses. Le plancher, à peu près carré, està environ 1,40 m a u dessus du sol, et il a 8 ou 9 mètres de côté. Une clôture légère, faite a vec des draps de lit suspendus à des barres de bois ou à une large corde tendue.

La scène se trouva it divisée en deux parties: l'une, la plus spacieuse, en a vant de la toile et constituant la scène proprement dite; l'autre en arrière et servant de coulisses aux acteurs. Les entrées se faisaient par les deux extrémités de cette espèce de rideau: quelquefo is on y ména gea it des ouvertures à cet effet, ma is le plus souvent on se contenta it d'en la isser à chaque bout flotter un pan mobile que l'acteur soulevait tout bonnement pour passer. Le souffleur avait sa place marquée dans la coulisse juste au point d'intersection des deux pièces de toile dont les bords étaient assez grossièrement cousus pour qu'il pûta u besoin a vancer le nez jusque sur la scène et se fa ire entendre de plus près a ux acteurs. Dans les ma nuscrits cette toile est a ppelée «la ta pisserie » (an ta pissiri) $;^{10}($...) on n'y employa it guère que des draps de lit (...) que l'on piqua it ça et là de quelques fleurs et que l'on enguirlanda it de verdures, branches de houx et rameaux de sapins. De décors il n'est ja ma is question. Et nous en a urons fini a vec l'installa tion ma térielle quand nous aurons dit que le plancher de la scène était percé d'une trappe par où les diables et leurs victimes étaient censées disparâtre dans les séjours infernaux (...). Ajoutons cependant qu'à côté de ce premier théâtre on en dressait parfois un second plus petit. (Le Braz, Histoire $d u$ théâtre celtique, pp. 474-5)

Quiconque a eu l'occasion de voir une pastorale souletine, encore de nos jours, peut constater que les éléments matériels structurant la scène des mystères bretons au XIXe siècle sont semblables à ceux utilisés dans les représentations souletines : draps de fond de scène séparant la scène des coulisses avec ouvertures sur les côtés pour les entrées

9 - On consultera notamment Le journal de route et les lettres de mission de F-M. Luzel publié en 1994. 10 - Dans les manuscrits de pastorales, le terme employé est tapiz 'tapis'. 
sur scène des acteurs; présence d'une trappe sur la scène destinée notamment à permettre aux diables à faire disparaître les cadavres des personnages négatifs ; absence totale de décors ; dans le passé utilisation parfois d'un seconde scène, plus petite, comme d'ailleurs dans Sa inte Elisabeth, où la mention de ce petit théâtre (tia tre chipia) apparaît pour le moins à deux reprises dans les didascalies. Il est évident que cette identité matérielle établit un modèle commun d'organisation de l'espace scénique des traditions bretonne et souletine. Elle se prolonge d'ailleurs par les parallélismes observés également dans la composition générique du répertoire, le fait de ne faire jouer que des hommes y compris pour les rôles féminins (en Soule, néanmoins, il existe aussi des tragédies uniquement jouées par des femmes), la manière de faire commencer et terminer la représentation par un prologue et un épilogue chantés selon les mêmes mouvements scéniques, l'utilisation dans la psalmodie de mélodies partiellement identiques. ${ }^{11}$ Aussi, comme rien ne permet de penser que les Souletins aient eu connaissance de la tradition bretonne, ni inversement, cette coïncidence ne peut s'expliquer que par le rattachement de ces deux traditions à un modèle, directement ou indirectement, commun. Tout ceci, au demeurant, ne doit pas masquer les différences, nullement négligeables, que l'on peut observer par ailleurs entre les deux traditions, qu'elles portent par exemple sur la technique de versification ou l'usage ou non de couleurs symboliques, sur l'emploi de personnages particuliers comme l'a nkou dans les mystères bretons, sur la coutume d'étaler la représentation des mystères sur plusieurs après-midi en Bretagne, ou encore sur le mode organisationnel associé aux représentations. Ceci témoigne simplement de la création dans chacune des régions d'un type particulier issu de ce modèle commun, adapté aux conditions et à l'histoire culturelle locales, ${ }^{12}$ comme il est de règle en ces matières.

L'appartenance historique des tragédies souletines à une tradition dramatique populaire largement répandue en France et au-delà, elle-même enracinée dans le théâtre médiéval, ne fait donc aucun doute. Ce lien a été relevé par les auteurs des premières descriptions de tragédies traditionnelles (par exemple, Michel 1857, p. 43, Vinson 1983, p. 309, Webster 1989, p. 244), et il a été souligné par Hérelle dont les travaux constituent la référence majeure dans l'étude de ce théâtre. La question reste ouverte cependant, de l'époque et des conditions dans lesquelles cette tradition est apparue et s'est fixée en Soule. Les réponses fournies ont été diverses, et l'un des objets de la présente étude est, non pas de répondre précisément à la question de la date de la création de la tradition des tragédies souletines (voir cependant une approximation au §10), mais de montrer que la documentation dont nous disposons à l'heure actuelle ne permet pas de remonter à une date précédant celle du manuscrit de Sainte Elisabeth, qui constitue la première référence connue relative à cette tradition particulière.

11 - Les airs basques sont notés et étudiés par Gavel (1911), les airs bretons par Quellien (1889, p. 279). N. Morel-Borotra (c. p.) m'a confirmé l'identité partielle de ces airs, très visiblement apparentés à la musique religieuse.

12 - Conditions certainement très différentes en Soule et en Bretagne, puisque les représentations des mystères sont attestées, de façon beaucoup plus précoce en Bretagne, y compris à travers l'impression au XVI ${ }^{e}$ siècle. Tel n'est pas le cas en Soule. La question de la continuité de la tradition dramatique bretonne entre les mystères anciens et ceux des XVIII ${ }^{e}$ et XIXe siècles se pose, compte tenu notamment de la quasi-absence durant une longue période de documents qui établiraient sans conteste cette continuité. Certains auteurs contemporains défendent l'idée d'un lien de filiation entre les deux traditions, en arguant de ce que les modes de versification caractéristique des mystères bretons anciens pourraient rencontrer des échos dans certains textes dramatiques bretons du XVIII et XIXe siècles (LeDuc 2001). Je m'abstiendrai évidemment de me prononcer sur le cas breton que je ne connais que de seconde main, et dont le contexte littéraire en tout état de cause est passablement différent en cette matière de celui du Pays Basque pour la période médiévale et post-médiévale. 


\section{§4. Le plus vieux manuscrit conservé.}

Le répertoire des tragédies souletines se caractérise, en ce qui concerne les textes, par le défaut total d'impression, ${ }^{13}$ lui même lié à l'absence de la notion d'auteur au sens moderne du terme, bien que les copies de manuscrits, au demeurant relativement nombreuses, eussent un propriétaire et fussent monnayables. ${ }^{14}$ Pour la période qui nous concerne, le répertoire concerne plus de 90 pièces, dont plus de 60 conservées à peu près intégralement (Hérelle 1926b, p. 9), et environ deux centaines de manuscrits (Hérelle 1928, p. 9). Il s'agit par conséquent d'un répertoire abondant, et même exceptionnellement abondant, compte tenu de l'histoire des textes en langue basque, en particulier dans le registre dramatique.

Premier point qu'il convient de souligner : parmi les manuscrits de ce répertoire, le plus ancien dont la date soit identifiable de manière sûre est précisément celui de Sainte Elisabeth de Portugal déjà rédigé, comme nous l'avons vu plus haut, pour une représentation donnée à Esquiule au tout début de 1750. Est-ce à dire qu'il s'agit du manuscrit conservé le plus ancien?

Ce n'est pas ce que nous dit Hérelle (1926b, p. 9 ; 1928, p. 9 et p. 134), qui mentionne à plusieurs reprises un manuscrit de Jeanne d'Arc, en son temps propriété de Clément d'Andurain, à propos duquel il offre la description suivante :

Le papier porte dans le filigra ne la da te de 1723, et l'écriture pa râ̂t être à peu près de la même da te (Hérelle 1928, p. 134).

La lecture de la date dans le filigrane conduisit Hérelle à considérer que le manuscrit le plus ancien conservé devait remonter à une date postérieure mais certainement proche de cette année (1723). Il donna même une reproduction de l'une des pages dans son catalogue analytique du répertoire du théâtre tragique (Hérelle 1928). Ledit manuscrit, depuis 1935, se trouve au Musée Basque, où il fut déposé par Mme de Malartic, sœur de Clément d'Andurain, et il y est donc consultable. Après avoir examiné ce manuscrit, à la fois incomplet et passablement dégradé, je m'étais positionné moi-même en faveur de l'hypothèse de Hérelle, en raison notamment de certaines particularités du manuscrit, interprétées de manière conjecturale comme pouvant représenter une marque d'ancienneté, en particulier le fait d'avoir des didascalies rédigées en français (Oyharçabal 1985, p. 57).

Désormais ces spéculations n'ont plus lieu d'être. La datation des supports matériels des documents a fait suffisamment de progrès pour que les techniques liées à l'analyse du papier (lieu de fabrication, nom du fabriquant, évolution de la réglementation juridique en la matière) soient employées utilement dans la datation des manuscrits et permettent d'éviter les erreurs dues aux datations déduites d'indices indirects souvent incertains. K. Segurola (2002) en a fait la démonstration en étudiant ce manuscrit de Jeanne d'Arc. Son examen lui a permis

13 - Evidemment, il existe avant 1914 quelques rares exceptions clairement dues au regard que des érudits ou certains bascophiles portèrent sur ce théâtre à partir du XIXe siècle. Vinson publia ainsi le texte d'une pièce traditionnelle (Sa int Julien d'Antioche en 1891), et en 1906, l'abbé Justin de Menditte et Clément d'Andurain publièrent Üskaldunak Ibañetan [Les Basques à Roncevaux], pièce s'inspirant de la tradition populaire, mais en réalité extérieure à celle-ci. Elle fut néanmoins jouée à Tardets en 1908 pour les Fêtes de la tradition basque (Hérelle 1928, 104).

14 - Dans la tradition, c'est l'instituteur ou régent (errejent) de la pastorale qui amène le texte (qu'il en soit auteur ou non), et qui organise toute la représentation dont il est régisseur, répétiteur, metteur en scène et souffleur. Il est le seul rétribué. F. Michel (1857, p. 46) par exemple indique qu'il recevait quarante francs au milieu du XIX ${ }^{\mathrm{e}}$ siècle, nourriture en sus. 
d'établir que la date de fabrication du papier de ce manuscrit est postérieure de près de trois-quarts de siècle à ce que pensait Hérelle, puisque la date portée en filigrane est 1793 (visiblement Hérelle avait lu 2 le chiffre 9, erreur parfaitement compréhensible eu égard à la qualité du papier et au dessin du 9, comme cela peut être constaté au regard de la reproduction des dates offerte par Segurola). Cette lecture est confirmée par l'identification des deux fabricants et du lieu de fabrication du papier. Le manuscrit de Jeanne d'Arc n'est donc pas le plus vieux manuscrit de pastorale conservé, loin de là. Par ailleurs, la rectification à laquelle conduit l'analyse de Segurola montre que les datations résultant d'interprétations d'indices secondaires -l'écriture pour Hérelle (1928, p. 134), les usages de rédaction des copies pour Oyharçabal $(1985$, p. 57)- ne sont que des pis-aller et peuvent aisément faire commettre des erreurs d'appréciation importantes dans les datations.

Le plus vieux manuscrit conservé en l'état actuel de nos connaissances est donc bien celui de Sainte Elisabeth de Portugal conservé au Musée Basque de Bayonne. La datation de 1750 pour ce manuscrit résulte tout simplement de l'indication du copiste dans l'ex-libris comme nous l'avons vu plus haut (\$2); en effet, l'usage constant dans les copies de tragédies souletines était d'indiquer dans l'ex-libris pour quelle représentation le manuscrit avait été établi. Observons cependant que dans la mesure où la feuille contenant l'ex-libris est probablement d'une seconde main, on ne peut exclure que le reste du manuscrit soit plus ancien. En ce cas, le régent Jauliber aurait conservé pour l'essentiel une copie préexistante, se contentant d'y rajouter les versets et informations paratextuelles de la dernière feuille.

Quoi qu'il en soit, nous avons donc ici une date borne certaine, démontrant qu'au milieu du XVIIIe siècle les tragédies traditionnelles étaient représentées en Soule. La lecture du manuscrit, qui est complet, ne laisse voir aucune indication pouvant donner à penser qu'il s'agissait d'une représentation à caractère exceptionnel. Ceci aurait sans doute été le cas si la copie avait été réalisée alors que la tradition était trop récente pour être connue par les spectateurs. Si nous admettons cette hypothèse, il en résulte qu'en 1750 cette tradition était suffisamment établie pour que, préparant la copie d'une représentation de tragédie à Esquiule, le copiste, dénommé Jauliber, ne fit aucune allusion ou remarque particulière sur la nouveauté que pouvait représenter le fait de faire jouer une tragédie en basque par les habitants.

Remarquons toutefois, même si l'argument est faible, que le texte du prologue et de l'épilogue de cette tragédie ne témoigne pas d'une lexicalisation dans la dénomination de ces représentations, ce qui indique, peut-être, mais il faudrait étudier ceci de plus près, une tradition relativement récente. En effet, le texte du prologue n'emploie que les vocables historia 'histoire' et errepresentazione 'représentation' pour désigner d'une part le thème de la tragédie, et d'autre part le spectacle dans son ensemble (orthographe modernisée) :

Zien $p<$ res> entziala huna niz / historia egiazko batez / Zier mintza tü nahiz 'Je suis venu devant vous /afin de vous parler /d'une histoire véritable')

hori dateke jente hunak / Gure errepresentazionia // eta galtho egiten dügü / zien atentzionia 'Ceci sera, bonnes gens, notre représentation //et nous vous demandons /votre attention' 
Ceci cependant ne concerne que le texte destiné à être déclamé. ${ }^{15}$ Les mentions paratextuelles du manuscrit, lesquelles sont en français, comme c'était l'usage habituel dans les manuscrits de ce théâtre traditionnel, font en effet apparaître, comme on l'a vu ci-dessus, la dénomination de tragédie (Fin de la tragedie de Ste Elisa beth). Il s'agit-là, d'une des principales appellations utilisées pour désigner les pièces de ce répertoire dans les copies de manuscrits, avant que celui de pastorale ne s'impose. Observons toutefois, que même s'il n'apparaît nulle part dans le manuscrit de 1750, ce dernier terme est déjà employé au milieu du XVIII siècle dans la tradition souletine car il figure par exemple dans le paratexte d'une copie de Jean de Paris datant de 1760 :

A Larrau le 25 juin 1760, il a esté joué la presente pastouralle. Fa it la presente coppie par moy Pierre d'Arhex, cordonier (mss. 19 du Musée Basque) ${ }^{16}$

Si les manuscrits conservés les plus anciens datent du milieu du XVIIIe siècle, la tradition des études sur ce thème a souvent mentionné des manuscrits non conservés plus anciens, et ces éléments sont fréquemment repris dans les études relatives aux tragédies souletines, sans qu'ils soient toujours accompagnés des avertissements utiles.

A propos des manuscrits non conservés, et plus généralement des textes anciens rapportés, il convient en effet de se prémunir par un examen critique attentif de possibles causes d'égarement. Parmi celles-ci, les plus classiques sont la supercherie littéraire et, évidemment, l'erreur de lecture. Ce sont là deux cas de figures que l'on rencontre dans la tradition des études sur le théâtre traditionnel basque, la première à propos de la pièce intitulée Marie de Navarre, et également, nous le verrons au §6, dans une configuration différente, à propos de Sa inte Geneviève.

\section{§5. Le manuscrit inventé de Marie de Navarre.}

§5.1. Il n'y a pas à ma connaissance de faux manuscrits proprement dits dans l'histoire des tragédies souletines. C'est-à-dire que nul ne s'est essayé à présenter un manuscrit qu'il avait fabriqué comme représentant un document plus ancien. Autrement dit, aucun des manuscrits existant ne résulte d'une création artificielle destinée à quelque escroquerie littéraire. Ceci dit, il y a bien eu des tentatives de tromperies littéraires, mais les faux auxquels ils ont donné lieu furent en quelque sorte virtuels, leur instigateur ayant dit avoir vu et copié un manuscrit ancien, sans jamais le montrer.

La principale tromperie littéraire dont il est question ici, et qui a été dévoilée par Hérelle, ${ }^{17}$ ne porte pas à vrai dire sur la tradition des tragédies souletines proprement dites, mais sur l'existence d'une poésie dramatique ancienne en langue

15 - Les études sur les pastorales ont toujours eu quelque difficulté à qualifier le mode d'expression des acteurs des tragédies (en dehors du prologue et du chant de l'ange pour lesquels la désignation de récita tif est employée usuellement) : les verbes décla mer ou psalmodier sont souvent utilisés. Hérelle (1928, p. 68) emploie aussi le verbe parler, qui est d'ailleurs celui que l'on trouve en basque dans les didascalies des manuscrits $(\operatorname{mintza})$, en indiquant qu'en dehors du prologue et de l'épilogue, on ne chante pas. V. Alford (1951) critique cette conception, qui résulte probablement du caractère, souvent qualifié de monotone, de l'air employé. Mais il s'agit pour Alford d'un récitatif chanté, dont elle fournit la notation (avec certaines variantes), selon ce qu'elle avait entendu à Alos en 1928 ; voir aussi Gavel 1911.

16 - Ce manuscrit avait été répertorié par Hérelle (1928, p. 117) et il est également étudié dans la thèse consacrée à cette tragédie par Mozos (1994, pp. 9-11), qui offre également une photocopie de la page de l'ex-libris, p. 455). Hérelle rapporte dans ses notes conservées à Bibliothèque municipale de Bayonne (mss. 111, doc. 23), l'observation de son collaborateur L. Irigaray signalant que le terme de pastorale figure également dans le texte de l'épilogue ; (voir aussi Hérelle 1926b, p. 83) 17 - Voir en particulier Hérelle 1926b (p. 88-89). 
basque. Elle fut mise en place en 1841, par Duvoisin, à une époque où les pastorales souletines n'avaient pas encore été bien étudiées, et où notamment la question de leur rattachement à une tradition dramatique plus ancienne et géographiquement plus large en Pays Basque se posait. W. Webster (1889) illustrait encore un demisiècle plus tard cette idée :

C'est depuis un temps comparativement récent que les pastorales ne sont jouées que dans la Soule. Autrefois, et même au dix-huitième siècle, on les jouait dans les autres parties du pays Ba sque frança is (W. Webster 1889, p. 243, note 1).

En 1841, Duvoisin avait publié dans l'Album pyrénéen, des extraits d'une représentation dramatique anonyme intitulée Marie de Navarre, mettant en scène des personnages de l'histoire navarraise remontant, estimait-il, au 10e siècle, et écrite en labourdin. L'objectif de Duvoisin lors de l'invention de ce faux a bien été résumé par Hérelle (1928) :

Cette pièce, écrite en dia lecte la bourdin et selon une métrique que l'on ne retrouve dans a ucune pastorale, est une supercherie littéraire. Le capita ine Duvoisin l'a fabriquée pour faire croire que le Labourd a eu, comme la Soule, un théâtre tragique, et pour attribuer a ux Labourdins, ses compatriotes, la fausse gloire d'avoir composé instinctivement des tragédies "régulières" (Hérelle, Le répertoire du théâtre tra gique, p. 130)

Ce propos de Hérelle peut toutefois prêter à confusion : Duvoisin, en réalité, ne prétend pas dans son article situer Marie de Navarre dans la tradition des tragédies souletines, à propos desquelles il ne dit rien et qu'il ne connaissait probablement pas à cette époque. ${ }^{18}$ Le modèle que Duvoisin avait à l'esprit pour fabriquer son faux était tout simplement le théâtre classique français. Il soulignait, en effet, que les pièces basques se rapprochent beaucoup des tragédies françaises, et il indiquait, à propos de la division en scènes et actes qu'il suit dans sa présentation de la pièce basque, qu'elle était implicitement présente dans le manuscrit qu'il prétendait avoir utilisé, bien que formellement non marquée :

Le manuscrit que j'a $i$ sous les yeux n'indique pas la division des actes et des scènes; je ne pense même pas que les Basques a ient ja ma is fa it de ces sortes de ces distinctions; ma is cette division se forme naturellement dans le cours du récit, et ici elle se trouve d'une régula rité remarquable (Duvoisin, Poésie dra ma tique des basques, Album Pyrénéen, mars 1841, p. 92 ; voir aussi F. Michel, in Le Pays Basque, p. 75)

§5.2. En fait, comme le fait observer Léon $\left(1909\right.$, p. 19), ${ }^{19}$ si le répertoire dramatique labourdin ancien imaginé par Duvoisin avait présenté les traits qui lui sont donnés dans Ma rie de Navarre, et que ce théâtre avait réellement existé, il n'aurait pas appartenu à la tradition des tragédies souletines traditionnelles, car les divisions et la régularité na turelle dont parle Duvoisin, et à travers lesquels il présente la pièce, n'a pas de sens dans le cadre de ce répertoire qui ignore totalement les conventions du théâtre classique français.

Ce faux reçut au cours du XIXe siècle des cautions savantes ou érudites, que Duvoisin parvint d'autant plus facilement à tromper que l'on ne doutait guère à l'époque que les tragédies souletines étaient l'expression d'une tradition très

18 - Al'inverse Duvoisin avait certainement déjà assisté à une représentation de tobera-munstra (théâtre charivarique). Il en fournit une description dans un autre article, intitulé la comédie des Basques, publié dans l'Album pyrénéen en 1841, sans jamais y évoquer les tragédies souletines, lesquelles, il est vrai, sont fort différentes à beaucoup d'égards.

19 - Dans sa thèse, Léon (1909, p. 18-19) n'ose évoquer l'hypothèse de l'escroquerie, mais relève bien que Marie de Navarre ne saurait appartenir au théâtre populaire ; voir infra note 29. 
anciennement présente en Pays Basque, et de manière plus étendue. Cette mise à jour venait à la fois confirmer et renforcer cette croyance. Les esprits étaient prêts, et Michel (1857, pp. 75-85), avant Webster, compta parmi les victimes de Duvoisin, dont il reprit les indications dans son ouvrage classique sur le Pays Basque en les rapportant aux tragédies souletines, pourtant fort éloignées à tous points de vues du modèle fabriqué par Duvoisin. Hérelle (1928, p. 89), dont les travaux sont beaucoup plus tardifs, ne fut pas victime de la supercherie, car le fait que la pièce fut structurée conformément au dispositif du théâtre classique français avait entre autres choses attiré son attention, et fait naître sa suspicion. De plus, ses soupçons furent avérés par suite de certaines confidences ; il lui fut en effet rapporté que Duvoisin avait avoué sa mystification à quelques basquisants. ${ }^{20}$

Hérelle estimait que Michel se doutait de la supercherie de Duvoisin au moment de la rédaction de son ouvrage, mais il n'indiquait par les raisons de cette supputation. ${ }^{21}$ Peutêtre lui avait-elle été inspirée par le fait que dans son ouvrage Michel s'attachait à présenter l'analyse Ma rie de Na va rre à travers de longues citations reprises de Duvoisin ? Pourtant, la suggestion de Hérelle est, à mes yeux, tout à fait improbable. L'échange de lettres qui eut lieu entre Duvoisin et Michel en 1855, près de quinze ans après la parution des extraits de Marie de Navarre, et qui a été publié par Dubarat dans la RIEB (1907, pp. 49-53), ne révèle pas une telle suspicion. Au contraire : Michel y prévient Duvoisin de son intention de reprendre son analyse de Marie de Navarre dans sa propre étude, et visiblement il lui fait entièrement confiance, s'en remettant aux renseignements qu'il voudra bien lui communiquer. L'emploi systématique des citations dans cette partie de son ouvrage ne montre pas une méfiance de Michel à l'égard de sa source, mais le souci de respecter celle-ci, qu'il tient visiblement en grande estime. ${ }^{22}$

\section{§6. L'improbable actrice de Sainte Geneviève dans l'oratoire.}

§6.1. On peut même constater que les indications supplémentaires données dans sa réponse par Duvoisin confortèrent Michel dans sa croyance en l'authenticité d'un autre texte, qui constitue très probablement une autre supercherie littéraire. Il s'agit d'une pièce intitulée Geneviève dans l'ora toire que Michel (1854 et 1857, p. 47-48), suivi plus tard par Hérelle (1928, p. 114), assimile à la pastorale Sa inte Geneviève de Brabant. ${ }^{23}$ Selon le brouillon du courrier mentionné précédemment, dont les propos sont repris en citation par Michel dans son Pays Basque (1857, p. 48), Duvoisin avait expliqué comment il avait recueilli quelque 25 ans plus tôt un chant des lèvres d'une

20 - Selon Hérelle, Duvoisin n'a pas toujours été a ussi discret dans les conversations particulières : car un notable basquisant de Bayonne a recueilli, dit-on, de la propre bouche du soi-disant éditeur l'aveu de cette regrettable paternité littéraire (Hérelle, 1926b, p. 89).

21 - Voici ce qu'indique Hérelle : Il convient pourtant de rappeler que Fr. Michel, sans mettre expressément en doute l'a uthenticité de Marie de Navarre, se défia it un peu des affirmations de Duvoisin en ce qui concernait les prétendue tra gédies la bourdines (Hérelle, 1926b, p. 89, note 2).

22 - Dans sa lettre du 27 mai 1855, il lui annonce son intention d'utiliser son article de 1841 : Indépendamment des déta ils généra ux sur le théâtre ba sque, je compte donner l'a nalyse de la pastorale de Clovis, dontj'ai eu une copie ancienne et celle du drame de Marie de Navarre que vous avez publiée (RIEB 1907, p. 50, souligné par BO).

23 - Michel avait déjà utilisé ces vers de Duvoisin dans une Lettre à M. P. Mérimée sur les représentations dramatiques dans le Pays Basque, parue dans l'Athenœum français du 9 décembre 1854, reprise dans le Messager de Bayonne du 14 décembre 1854. Il n'indiquait pas sa source, évoquant simplement un chant tiré de la pastorale de sa inte Geneviève, dont il offrait des vers traduits, et le tout début en basque. Lorsque Michel prit contact par lettre avec Duvoisin pour lui demander une collaboration, ce dernier se montra surpris d'avoir vu les vers de Geneviève dans l'ora toire connus du premier, et revendiqua d'en avoir été le collecteur: J'ai lu avec le plus vif intérêt votre lettre à M. Mérimée sur les représentations dramatiques des Ba sques. J'y a vu, non sans étonnement, la traduction d'un morcea u dont je croya is bien être le seul possesseur, Geneviève dans l'Oratoire. Je l'a i recueilli, il y a 25 ans, de la bouche d'une pa uvre vieille fille ... (Réponse de Duvoisin à la lettre de Michel en date du 27 mai 1855, RIEB 1907, p. 53). J'ignore dans quelles conditions Michel avait eu connaissance de ces vers prétendument recueillis par Duvoisin. 
vieille fille (voir la citation de la note 23 , et la description édifiante de cette informatrice plus loin dans ce paragraphe), dont la raison avait été ébranlée à la suite du succès qu'elle avait connu en jouant le rôle de sainte Geneviève dans sa jeunesse. Là encore, comme pour Marie de Navarre, les propos de Duvoisin furent interprétés par Michel comme si ces vers devaient être rapportés à la tradition des tragédies souletines, alors que le premier n'avait fourni aucune indication précise du lieu où il prétendait avoir recueilli ces vers, et le contexte de son courrier de 1855 laissant même penser que ladite actrice était labourdine. En effet dans sa lettre, le passage sur l'actrice ayant joué le rôle de Geneviève est suivi de l'indication suivante :

Les Labourdins ne connaissent pas, dites-vous, les représenta tions théâtrales. Il serait plus juste de dire qu'ils ont abandonné la tragédie, car il y a encore à Sa int-Jean-de-Luz desfemmes qui l'ont jouée (Brouillon de la lettre de 1855 de Duvoisin à Michel, RIEV1907; précision reprise sans indication de citation par Michel 1857, p. 53).

La probable supercherie, cette fois-ci, ne porte pas sur un manuscrit virtuel, mais sur un chant supposé appartenir à une tradition dramatique, que Duvoisin disait avoir recueilli oralement. En fait, ce dernier se plaçait dans une perspective semblable à celle qui l'avait poussé à inventer le manuscrit de Marie de Navarre presque quinze ans auparavant : il voulait montrer, en s'appuyant sur un témoignage certainement imaginaire, qu'il existait bien une tradition de poésie dramatique (titre de l'article de Duvoisin dans l'Album pyrénéen de 1841), laquelle aurait vécu suffisamment tard pour qu'il ait pu personnellement en recueillir un témoignage direct.

Pourquoi Duvoisin évoquait-il uniquement des actrices et non des acteurs ? La chose est curieuse. ${ }^{24}$ Il semble que pour lui la forme finale du vieux théâtre, dont il était convaincu de l'existence, correspondait à des représentations où les acteurs étaient tous du même sexe, et singulièrement féminins. ${ }^{25} \mathrm{Il}$ considérait, en effet, que la séparation des sexes dans le théâtre populaire basque résultait d'une évolution tardive, causée par les pressions du clergé, d'où serait né un répertoire fait uniquement pour les femmes. Les vers de Geneviève dans l'ora toire correspondaient ainsi dans sa conception à la phase ultime de ce théâtre populaire ancien, et il situait cette extinction (ailleurs qu'en Soule) à la fin du siècle précédent. ${ }^{26}$

24 - On ne peut absolument exclure qu'à la fin du XVIII e siècle certains membres féminins de la société luzienne aient pu s'adonner aux plaisirs des représentations dramatiques, peut-être dans le cadre d'un théâtre semblable à celui du théâtre des collèges (attesté au collège de Bayonne au siècle précédent), qui aurait été pratiqué dans des institutions éducatives pour jeunes filles. Cependant ceci n'est pas documenté. Quoi qu'il en soit, l'existence très hypothétique d'une telle pratique théâtrale dans ce contexte à la fin du XVIII siècle doit bien être séparée de la question de l'authenticité de Ma rie de Navarre et de Geneviève dans l'oratoire, et, évidemment, de l'existence en Labourd d'une tradition de théâtre populaire, parallèle à celle des tragédies souletines, comme voulait le faire croire Duvoisin. 25 - Dans les notes manuscrites de Duvoisin, on rencontre certains propos restituant sa conception de l'évolution du répertoire dramatique populaire ancien (dont il ne doutait pas de l'existence), en ce qui concerne la séparation des sexes: Il yeut un temps où les jeunes filles joua ient les rôles de femmes. Les désordres que ces accointances firent na ître servirent de texte à plus d'un sermon et le succès du clergé pour empêcher ces a malga mes l'emporta par le concours des parents, qui éta ient bien a ise d'épargner à leurs enfants les dangers d'un contact passionné. Alors on composa des pièces pour des jeunes filles exclusivement, et pour les ga rçons; et celles où les sexes éta ient mêlés furent jouées par les garçons qui s'a djoigna ient des jeunes gens imberbes propres à être dans les rôles de femmes (mss. 461 de la bibliothèque de Bayonne, 6e registre, p. 95).

26 - Les notes manuscrites de Duvoisin laissent apparaître sa contrariété de voir le répertoire populaire basque étudié principalement par des amateurs étrangers, dont il semble mettre en doute, sinon l'honnêteté, du moins leur respect du patrimoine littéraire basque : Que sont devenues ces pièces, car il y en a eu beaucoup et il s'en trouve très peu; encore qu'elles sont remaniées et déna turées par les copistes. Ce sont des a mateurs qui s'en sont emparés. (...) C'est a insi que les a mateurs [après avoir évoqué Badé et Michel] ont dépouillé le pays et dispersé les monuments de la poésie nationale, (mss. 461 de la bibliothèque de Bayonne, 6e registre, p. 95). Ces remarques acides et passablement injustes furent faites, soulignons-le à la décharge de leur auteur, en un temps précédant l'action de Hérelle, Vinson ou Webster, en vue de sauver l'essentiel de ce qui a été préservé du répertoire dramatique traditionnel. 
Les vers que Duvoisin prétendait avoir recueillis de son informatrice sont en labourdin, pas en souletin ou en bas-navarrais, et n'ont évidemment aucune similitude avec le mode de versification (quatrains de mesure libre et à rimes paires assonancées), employé dans les tragédies souletines.

Michel, qui n'était pas en mesure de séparer la tradition des tragédies souletines d'un répertoire dramatique basque ancien, dont il était convaincu de l'existence nécessaire, et dont Duvoisin portait le témoignage, assimile les vers de Geneviève dans l'oratoire au théâtre souletin, et concrètement à la pastorale de sainte Geneviève (1857, p. 47). Pourtant, tant les différences dialectales que la dissemblance entre les modes de versification ou le contenu des textes rendent cette assimilation des vers de Geneviève dans l'ora toire au répertoire traditionnel souletin dénué de la moindre vraisemblance.

Voici par exemple le début du texte basque et sa traduction, tels que présentés par Michel :

$\begin{array}{ll}\text { Nic ez dioterran, } & \text { Je ne puis dire } \\ \text { Nic ez diot pentza (sic) } & \text { je ne puis penser } \\ \text { Bihotzean zerdudan. } & \text { ce que j'ai au cœur. } \\ \text { Oi! Neure hatsa } & \text { Ah ! Le souffle de ma vie } \\ \text { Doidoia badoha } & \text { à peine s'élève } \\ \text { Airera } & \text { dans l'air } \\ \text { Ceruetara : } & \text { Vers les cieux; } \\ \text { Neroni banoha } & \text { moi-même je m'en vais } \\ \text { Harekin batean } & \text { avec lui } \\ \text { Airean, etc. } & \text { dans l'air. } \\ \text { (F. Michel, Le Pays Basque, p. } & 48)\end{array}$

§6.2. Il est évident que l'on n'est pas en présence de versets semblables à ceux des tragédies souletines. Il est vrai que Michel parle au sujet de ces vers de 'chant', terme dont on ne sait s'il l'applique au mode de déclamation des versets de ces tragédies; voir note 15). On pourrait se demander alors, si l'on ne serait pas en présence, pour lui, d'un texte particulier dérogeant au mode de composition habituel. L'argument toutefois ne tient pas. En effet, les seuls chants spécifiques apparaissant dans les tragédies souletines traditionnelles sont des chants liturgiques classiques (par exemple, le Te Deum dans Sa inte Elisabeth) et, très rarement, des chants profanes eux aussi connus; il n'y a pas de chants originaux se rapportant directement à la tragédie (voir Hérelle 1922, p. 306). Michel considère d'ailleurs que la seconde partie des vers qu'il cite, extraits de Geneviève dans l'oratoire, correspond à un chœur d'anges. Or le type de composition utilisé à cette occasion est identique à celui rapporté par Duvoisin dans les versets précédents, et n'a aucun rapport avec celui employé pour les vers des anges des tragédies souletines (composés également en versets comme pour les autres rôles, même si l'air du récitatif est différent).

Les clichés qui caractérisent le témoignage de Duvoisin font songer à une reconstruction littéraire réalisée de façon caricaturalement conforme à l'imagerie romantique ; voici en effet le portrait qu'il nous fait de l'actrice occasionnelle, des lèvres de qui il prétendait avoir recueilli ces vers :

Je l'ai connue grande, maigre et noire; elle avait les yeux vifs et d'une sévérité extraordinaire, quand elle ne murmurait pas ses chants devant les rochers autour 
desquels, la quenouille au côté, elle fa isa it paître quelques misérables brebis. Il me fallut plusieurs mois de patience et de cour pour me faire agréer de la pauvre monomane; encore, n'ai-je pu obtenir d'elle que la plus faible partie de ce qu'elle savait. Ma is j'a $i$ vu cette femme ridée, un genou sur un escabea u, la figure à moitié tournée vers le public devant lequel elle se supposait, les yeux fixés au mur sur une image de Juif errant qui remplaça it le crucifix de l'ora toire; sa poitrine se gonfla it comme a ux bea ux jours de sa jeunesse; j'a i entendu sa voix chevrotante, vibrante, pénétrante, et jamais je n'oublierai cette scène. (Lettre de Duvoisin à Michel rapportée par ce dernier dans Le Pays Basque, p. 48 , et correspondant au brouillon d'une lettre datant très certainement de 1855, publié par Dubarat dans la RIEB, 1907, p. 53).

Le texte parle par lui-même. Cette bergère obnubilée, chantant dans un murmure, au milieu de quelques brebis, les vers qui lui rappelaient les succès de comédienne occasionnelle ayant illuminé sa jeunesse, est évidemment un stéréotype littéraire. On pourrait supposer que pour évoquer une bergère labourdine Duvoisin se placerait dans une position de proximité, ainsi d'ailleurs que l'en priait Michel, qui lui demandait de lui brosser le portrait de ses compatriotes contrebandiers. ${ }^{27}$ Mais un tel positionnement l'eût conduit d'une certaine façon à une trahison à l'égard d'une histoire qui ne pouvait être simplement celle qui aurait résulté d'un témoignage authentique (c'est-à-dire constatant l'absence d'attestation hors de Soule, particulièrement en Labourd, d'une poésie dramatique basque conforme aux représentations historiques en vogue en un temps largement dominé par les conceptions romantiques). Dans la représentation que lui même, comme la plupart des érudits et folkloristes de ce temps, avait du passé culturel du Pays Basque, il était impossible que les Basques n'eussent pas développé une poésie dramatique. ${ }^{28}$ La singulière bergère de sa relation (avec en écho une évocation, sans doute non fortuite, $\mathrm{du}$ monde pastoral) lui permettait de déguiser un pieux mensonge, expression d'une certitude fondée sur une méta-vérité. Sinon, puisque la poésie dramatique s'était perpétuée en Labourd jusqu'à la fin du XVIIIe siècle, pourquoi Duvoisin, né en 1810 à Ainhoa et ayant été élevé à Espelette, en plein cœur du Labourd, aurait-il eu besoin de recourir au difficile témoignage d'une pastourelle insolite, bien que littérairement très convenue, à propos de laquelle il n'indique (et pour cause) ni le nom, ni le lieu où elle avait vécu ? Et tous les autres contemporains de cette actrice avaient-ils perdu la mémoire, au point que nul autre n'aurait été en mesure de recueillir l'écho de tels souvenirs en cette première partie du XIXe siècle où ces questions sont pourtant en vogue ?

§6.3. La critique moderne n'a guère prêté attention à ce témoignage sans doute imaginaire de Duvoisin. Il est vrai que les textes apocryphes basques ont surtout été étudiés à travers les chants hérö̈ques fabriquées (Chant d'Altabizkar, Chant d'Annibal, Chant d'Abarka, ...), ou les légendes comme celle d'Aitor, créée par

27 - Telle était en effet l'information que Michel avait demandée en premier lieu à Duvoisin : On voit combien les choses de votre pays vous sont fa milières et à quel point il vous est a isé de les rendre avec bonheur. Un morcea u sur les contrebandiers des Basses-Pyrénées, de la partd'un homme comm is [Duvoisin était officier des douanes] pour les observer, sera it sans prix; so it dit sans faire tort a ux a utres ma tières qui se rapportent à mon travail (Lettre de Michel à Duvoisin du 17 mai 1855, RIEB 1907, p. 50)

28 - Dans ses notes manuscrites à propos de l'article de Bladé (1866), Duvoisin observe : Je doute que les Basques a ient écrit des épopées comme il s'en trouve en langue d'oc. S'il en a existé en basque, elles ne sont pas parvenues jusqu'à nous. Ma is un fait certain, c'est que nos traditions guerrières ont fa it longtemps les délices du peuple dans les spectacles qui se donnaient chaque année, dans une ou plusieurs localités du pays. Les tragédies basques écrites en vers étaient [illisible] sur les places publiques par les jeunes gens de la commune (mss. 461 de la bibliothèque de Bayonne, 6e registre, p. 95, texte souligné par BO). Voir également au $\S 8$ le témoignage très explicite de l'archiviste contemporain J.AC. Buchon. 
Chaho, en raison de leur retentissement dans l'interprétation du passé lointain des Basques au XIXe siècle (Bladé 1869, pp. 444-482, Vinson 1984, pp. 29-56, et plus récemment Juaristi 1987, ch. IV-V et Bidart 2001, pp. 206-218). Les textes apocryphes du registre théâtral basque, à l'inverse, probablement en raison de leur caractère purement littéraire, n'ont guère retenu l'attention de ces auteurs. Vinson, par exemple, n'évoque pas le cas de Marie de Navarre, dont on peut penser pourtant qu'il en pressentait, ou connaissait, l'origine. En effet, il ne mentionne pas cette pièce parmi celles appartenant au répertoire dramatique, bien qu'il cite dans ses références bibliographiques les travaux où la pièce est présentée et analysée. Même A. Léon (1909, pp. 18-19), qui avait bien repéré le caractère non traditionnel de cette pièce, s'abstint d'évoquer clairement l'hypothèse d'un faux littéraire dû au découvreur. ${ }^{29} \mathrm{C}^{\prime}$ est finalement Hérelle (1926b, pp. 88-89) qui clarifia définitivement les choses de manière publique.

L'authenticité des vers de Geneviève de Brabant par contre ne fut contestée par quiconque. Hérelle, pourtant au fait du faux que constituait Marie de Navarre, s'abstint d'en mettre en doute l'authenticité bien qu'ils provinssent du même auteur. On aurait pu s'attendre également à ce qu'il contestât leur attribution par Michel à la pastorale Sainte Geneviève de Brabant, qui appartient bien, elle, au répertoire des tragédies souletines. Mais ne sachant pas le basque et n'ayant pour seule source que les indications de Michel, Hérelle reprit dans son répertoire cette assimilation, sans que les vers de Geneviève dans l'ora toire ne correspondissent en aucune façon au code textuel, pourtant bien typé, des tragédies souletines.

Les historiens de la littérature basque, pour la plupart, n'ont pas évoqué ces vers, il est vrai très incomplètement connus dans leur version basque jusqu'à une date récente. Ils ne figurent pas, par conséquent, dans la plupart des chansonniers ou des recueils ou anthologies poétiques, en dehors, évidemment, de l'ouvrage de Michel (1857) qui les fit spécialement connaître. A ma connaissance, la seule exception est Garzia \& Urkizu (2001, p. 88-89 et 857$){ }^{30}$ qui publient pour la première fois le texte basque en entier en y voyant un poème extrait d'un drame anonyme joué en Labourd vers 1780. Ils suivent donc les indications de Duvoisin quant à l'origine prétendument anonyme des vers et, apparemment ils en déduisent également la datation (vers 1780). En effet, selon les indications d'Urkizu dans Historia de la litera tura va sca (Urkizu \& al. 2000, p. 380) ; il n'apparaît pas que le manuscrit source utilisé pour leur édition mentionnait cette date. Remarquons que ces auteurs évitent la confusion entre Geneviève dans l'ora toire et le répertoire des tragédies souletines traditionnelles et notamment Geneviève de Brabant. Ajuste titre, car nulle part Duvoisin ne propose une telle assimilation, qui est due à une confusion de Michel, non repérée et, par conséquent, confirmée ensuite par Hérelle. Mais il convient d'aller plus loin, et de questionner le témoignage de

29 - La formulation de Léon, qui n'était pas dupe, évite une dénonciation précise : Ma is à moins de supposer a ux Labourdins de cette époque une technique dra ma tique toute différente de celle des Souletins, il est difficile de ne pas voir, jusqu'à plus a mple informé, dans le drame dont je parle [Marie de Navarre] plutôt le passe-temps de quelque lettré qu'un spécimen authentique du théâtre basque proprement dit (Léon, Une pastorale basque: Hélène de Constantinople, p. 18, texte souligné par BO).

$30-\mathrm{Qu}$ 'il s'agisse très vraisemblablement d'un texte apocryphe, au même titre que Marie de Navarre ou que le chant de l'Altabizkar, ne justifiait pas, évidemment, qu'il demeurât inaccessible dans sa plus grande partie (contrairement, nous l'avons dit, à sa traduction française offerte par Michel dans son Pays Basque). On regrettera cependant certaines erreurs que l'on devine dans l'édition de ces vers. Par exemple, on y trouve l'extrait suivant: Zuri esan eztia / Heda / Diezadazula. Ceci ne veut rien dire, alors que Michel dans sa publication de 1857 offrait de ce passage une traduction limpide : étendez sur moi votre douce main. Il convient probablement de corriger le texte basque en Zure esku (<escu ?) eztia. Ce n'est pas le seul cas où la comparaison des textes basque et français donne à penser que le texte édité devrait être rectifié. 
Duvoisin lui-même : le caractère apocryphe de ces vers semble en effet plus que probable, si l'on tient compte du contexte historico-littéraire et des circonstances dans lesquelles ils apparaissent et nous sont rapportés.

\section{\$7. La datation rapportée (1634) de l'ex-libris du manuscrit de Saint Jacques.}

§7.1.Comme indiqué plus haut, les manuscrits de pastorales, lorsqu'ils sont complets comportent toujours une mention paratextuelle indiquant pour quelle représentation la copie fut établie, ainsi que le nom du copiste-régent (et éventuellement celui de possesseurs ultérieurs). Par conséquent, la datation des copies manuscrites de tragédies traditionnelles ne laisse pas de place au doute, sauf bien sûr lorsque les copies sont incomplètes, comme dans le cas de la copie de Jeanne d'Arc du Musée Basque évoquée précédemment (cf. §4).

Imaginons maintenant le cas d'une date de représentation qui ferait apparaître une datation antérieure au manuscrit lui-même. Cela serait-il possible ? Théoriquement, c'est à dire sans tenir compte des usages des copistes dans la tradition souletine, cela serait évidemment possible : il suffirait, par exemple, que le copiste d'un manuscrit B, en établissant sa copie à partir d'un manuscrit $\mathrm{A}$ (nécessairement antérieur à $\mathrm{B}$ ), recopie sur le manuscrit $\mathrm{B}$ la date de représentation figurant sur la copie A

Si je pose cette hypothèse, c'est parce qu'elle a été avancée par Hérelle (1926b, p. 110, 1928, p. 46) à propos de la copie d'un manuscrit de Sa int Jacques, actuellement déposé à la Bibliothèque Nationale (ms. 211). Ce document, dont le copiste, Jean-Pierre Saffores, est connu, date du XIXe siècle mais ferait apparaître selon Hérelle la date de 18 août 1634, d'où l'hypothèse évoquée plus haut :

La copie du Saint Jacques de la Bibliothèque nationale, $n^{\circ} 211$, a été faite il y a une centaine d'a nnées seulement, et néanmoins on y lit, a près l'épilogue, la da te très lisible du «29 août 1634 ». Comment expliquer cette date qui ne peut être celle de la copie ? M. Omont, conservateur du département des manuscrits, avec l'autorité de sa grande expérience en ma ma tière, estime que cette da te a dû être reproduite par le copiste d'a près une inscription que porta it l'ancien ca hier sur lequel la copie nouvelle a été faite. (Hérelle, Les pastora les à sujets tra giques considérées littérairement, p. 110)

Hérelle connaissait bien le répertoire des tragédies souletines, et il savait que le manuscrit ne pouvait dater de 1634, puisque Saffores, le copiste du XIXe siècle, conformément à la tradition, s'identifiait. Ce dernier était même parfaitement connu, car sans conteste le régent de tragédies traditionnelles le plus célèbre en son temps, ainsi que le rapporte Chaho, qui dut le connaître fort bien, car tous deux étaient natifs de Tardets. ${ }^{31}$

L'hypothèse évoquée par Hérelle lui avait été suggérée, nous dit-il, par M. Omont conservateur du département des manuscrits de la Bibliothèque Nationale, qui faisait état d'une pratique fréquente dans les copies de manuscrits. Mais cette pratique, et cela évidemment ledit conservateur devait l'ignorer, était inconnue dans la tradition des copies de tragédies traditionnelles, qui n'offre aucun exemple d'un tel cas. Dans le contexte qui est le nôtre, la leçon proposée est donc très coûteuse, car elle modifie sensiblement ce que l'on sait des usages des copistes traditionnels et de l'esprit dans lequel ils travaillaient. Il faut par conséquent bien vérifier que cette hypothèse est matériellement justifiée.

31 - Urkizu (1990) a publié la tragédie de Saint Jacques d'après ce manuscrit dans sa totalité. Une partie (épisode du miracle des pendus) avait été auparavant été publiée par Oyharçabal (1991, p. 251-283). 
§7.2. Lorsque j'eus l'occasion de m'assurer par moi-même de ce point en consultant ce manuscrit à la Bibliothèque Nationale il y a environ vingt-cinq ans, je me convainquis rapidement du contraire. Contrairement à Hérelle, qui pensait, comme la plupart des auteurs jusque-là, mais en l'absence de toute base documentaire allant en ce sens, que la tradition souletine remontait au moins au XVIe siècle, je me situais dans un contexte différent, où on ne pouvait exclure d'être en présence d'une tradition plus récente. Les attentes induites par nos présupposés respectifs étaient probablement différentes et, en tout état de cause, l'apparition d'une datation aussi ancienne sur un manuscrit dont on connaissait très bien le copiste du XIXe siècle devait, pour être validée, reposer sur des bases incontestables. C'est la raison pour laquelle je souhaitais vérifier par moi-même les indications de Hérelle. L'examen du manuscrit me permit d'écarter la lecture du 6, qui apparaissait pourtant, dans un premier temps, comme s'imposant au regard ; voir Oyharçabal (1991, p. 251).

Les apparences peuvent être trompeuses, surtout lorsqu'elles paraissent confirmer des attentes. Le cas de la copie Saffores du manuscrit de Sa int Jacques est de ce point de vue très significatif. Le chiffre transcrit par le copiste est un 8 (année 1834 donc), mais avec un dessin tel que la boucle du bas du 8 est réduite à un point (en fait un croisement dans le tracé du signe), de sorte que dans un examen superficiel elle paraît absente. Al'opposé la boucle du haut du 8 est mal fermée, et dans une lecture superficielle paraît correspondre plutôt au dessin d'un 6 . Je pense, par conséquent, que seule une mauvaise lecture (soutenue par les attentes de datation s'approchant du XVIe siècle) explique les indications de Hérelle.

Cette lecture ayant été contestée par l'éditeur de cette tragédie (Urkizu 1990, p. 145 ; 1994, p. 26) et s'agissant d'une question factuelle, afin que chacun puisse se faire une opinion, je présente ci-dessous, après Oyharçabal (1999), une reproduction de la date contestée (Figure 2), et analyse ensuite la graphie du chiffre prêtant à discussions.

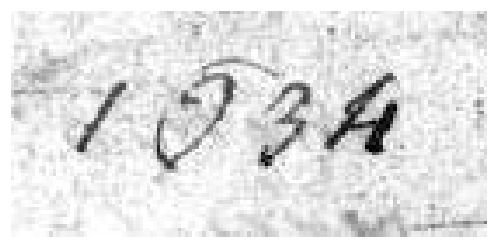

FIG.2. Copie de la date figurant sur le manuscrit :

Pour lire sans équivoque le chiffre 8 , et non 6 , il faut suivre le geste du copiste dans sa graphie. Pour cela, il convient de voir comment Saffores graphie ces chiffres dans des lectures indiscutables.
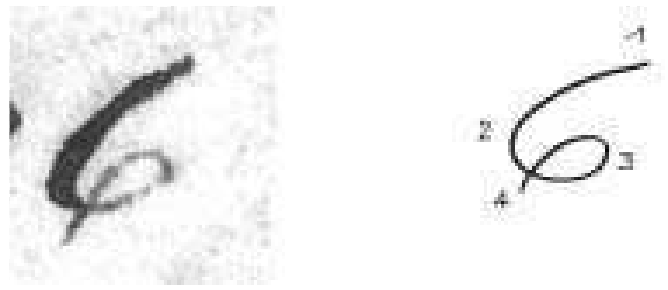

FIG. 3. Copie du chiffre 6 et tracé correspondant 
Considérons le chiffre 6 sur la figure 3 ci-dessus (à partir d'une copie réalisée sur le même manuscrit de Saffores). Il est tracé d'un seul geste, sans rupture dans le tracé, en partant du haut dans une orientation gauche (point 1), vers le bas (point 2), pour se diriger ensuite vers la droite en initiant une boucle vers le bas (point 3), laquelle boucle se ferme par le tracé qui se prolonge vers le bas (point 4).
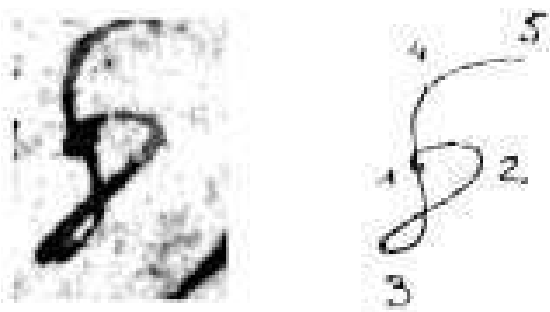

FIG 4. Copie du chiffre 8 et tracé correspondant

Examinons maintenant ci-dessus le chiffre 8 dans la figure 4 (copie du chiffre prise elle aussi sur ce même manuscrit de Saffores). Il est également tracé d'un seul mouvement. Bien qu'apparaissant au milieu du dessin, le point de départ est la boucle supérieure du 8 (point 1 ) ; le tracé se dirige après vers la droite (point 2) avant de redescendre vers le bas dans une orientation gauche, et repartir vers le haut (point 3) verticalement, ce qui permet de dessiner la boucle inférieure du 8. Le tracé va fermer la boucle supérieure et se poursuit ensuite au-delà de celle-ci vers le haut (point 4), pour se terminer par une large courbe orientée à droite (point 5).
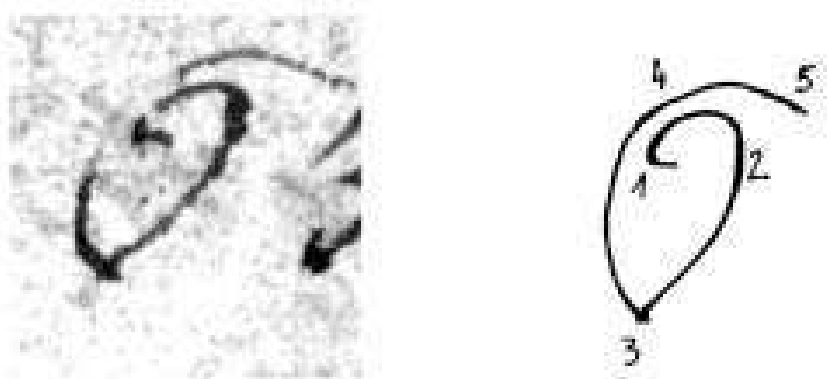

FIG. 5. Copie du chiffre contesté et tracé correspondant

Enfin, considérons maintenant le chiffre contesté apparaissant dans la figure 5. Il est réalisé d'un seul geste. Le point de départ est au centre du dessin (point 1). Le tracé part vers la droite (point 2) pour redescendre vers le bas dans une orientation gauche, avant de remonter en courbe vers le haut à la suite d'un croisement correspondant à un renversement dans le tracé (point 3). La ligne va au-delà du point de départ (point 4) et se termine par une large courbe orientée à droite (point 5)

En fait, comme le montre la décomposition du geste, le tracé de la figure 4 correspond exactement à celui de la figure 3, à la seule différence que la boucle inférieure (point 3 ) est réduite au minimum dans le premier cas, c'est-à-dire à un renversement d'orientation dans le tracé, qui forme comme un point de croisement. Mais il est évident, du moins à mes yeux, que l'on est en présence dans 
les deux cas d'un même tracé, correspondant effectivement à un 8 , comme il est normal s'agissant d'un manuscrit du XIXe siècle.

Les conclusions que l'on tire d'un tel examen me paraissent peu contestables, et il est difficile de leur opposer une interprétation qui ferait de ce document une copie d'un manuscrit du XIXe siècle plus ancien de deux siècles. La mention d'exlibris de tragédie la plus ancienne qui soit attestée est donc dans notre vue celle de Sa inte Elisabeth.

\section{$\S 8$. Le cas du manuscrit de 1500 acheté par Buchon}

§8.1. Parmi les arguments avancés en faveur d'une datation ancienne de la tradition des tragédies souletines, et fondés sur la date de manuscrits perdus, l'un repose sur un témoignage unique datant de 1839. Il apparaît dans un article publié dans le Mémorial des Pyrénées par un archiviste, J. A C. Buchon. La formulation la plus nette de cet argument a été exprimée par Hérelle (1926b, p. 110) :

Buchon qui, en sa double qualité d'inspecteur général des Archives et des Biblio thèques, et de savantéditeur d'un grand nombre de chroniques fra nçaises et étrangères, éta it très bon juge en paléographie, dit a u sujet des manuscrits de pastorales qu'il a vues en 1839 chez Saffores, à Tardets; "L'une (de ces pastorales), intitulée Clovis, est certainement un manuscrit de 1500, et je le lui a i a cheté.»Malheureusement cette copie de Clovis est perdue ; ma is pour ce qui concerne l'âge du manuscrit, nous pouvons en croire Buchon sur parole (G. Hérelle, Les Pa storales à sujets tragiques considérées littérairement, p. 110).

Comment faut-il évaluer le témoignage de Buchon, qui, en tant qu'archiviste, devait effectivement posséder une compétence certaine en matière de datation des manuscrits ? Faut-il notamment le croire sur parole, dès lors que ce manuscrit, qu'il disait avoir acheté, n'a pas été retrouvé, et que nul autre ne l'a jamais eu entre les mains?

La réponse, contrairement à ce que propose Hérelle (1926a), ne saurait être simplement positive au motif que Buchon, collecteur et éditeur des Chroniques nationales françaises $d u$ XVe et XVIe siècles et Inspecteur général des Archives et Bibliothèques de France, était compétent en matière de paléographie. Certes son témoignage doit être pris en compte, en particulier s'il vient appuyer d'autres indications moins virtuelles allant dans le même sens. Mais isolé, et dans une large mesure contraire à ce que l'on sait par ailleurs de la matière, la valeur d'un témoignage de cette nature est très amoindrie.

Les études basques ont fait depuis plus d'un siècle et demi suffisamment de progrès pour que l'on mesure la portée de certaines conjectures : l'existence au XIXe siècle d'un manuscrit de représentation dramatique en langue basque datant de l'an 1500 représenterait quelque chose de proprement exceptionnel, et avant d'avaliser un témoignage isolé tel que celui de Buchon (assurant de cette existence sans que le document ait été attesté par quiconque, autre que lui-même), il est nécessaire, pour le moins, de procéder au préalable à un examen critique sans complaisance à son sujet.

§8.2. Le premier élément qu'il convient de considérer est celui relatif à l'époque où ce témoignage est formulé. Il s'agit de la période de développement des études consacrées aux textes et traditions populaires anciennes, notamment dans les régions périphériques à forte personnalité comme le Pays basque et la Bretagne en 
France, et en bien des endroits le mouvement s'accompagne de la fabrication de textes remaniés ou apocryphes, fausses légendes et coutumes inventées. ${ }^{32}$ En Bretagne, cette même année 1839, La Villemarqué publie un recueil de poésies populaires, le Barzaz Brez, dont les textes sont largement remaniés. En Pays Basque, Garay de Monglave quatre ans plus tôt s'était essayé à une contrefaçon, inspirée des poésies ossianiques, en faisant publier le chant de l'Altabizkar, Chaho émaillait ses écrits d'observations dues aussi bien à son imagination fantasque et luxuriante (et simplement faussaire aussi, à l'occasion) qu'à son esprit curieux et original, et nous avons déjà évoqué la contribution plus que suspecte de Duvoisin à propos des reliquats d'un ancien théâtre basque en Labourd, avec Marie de Navarre et Geneviève dans l'ora toire.

Est-ce à dire qu'il faille considérer Buchon comme un escroc littéraire lorsqu'il mentionne un manuscrit certainement de l'an 1500 acheté chez Saffores ? Pas nécessairement, mais il est utile de rappeler le climat du temps, qui influence le regard que les contemporains portent sur les productions de la littérature populaire, notamment lorsque celle-ci est linguistiquement exotique. Ceci transparaît d'ailleurs dans le témoignage de Buchon. Son propos se présente comme une lettre adressée à Dumas depuis Tardets, par un savant venu en Pays Basque en quête d'exotisme et de trésors littéraires transmis en dehors des circuits de la culture savante :

Il me semblait vraiment extraordinaire qu'un peuple vif et spirituel, vain de sa na tiona lité, pourou d'une langue restée à peu près la même depuis plus de deux mille ans, d'une langue qui n'a a ucune sorte d'a na logie avec les a utres langues de l'Europe, d'une langue savante dans ses combina isons grammaticales, et douce dans ses inflexions, n'eût ja ma is possédé un seul homme qui comme Burns en soignant sa petite ferme, comme le berger d'Eltrick (sic) en gardant ses bestiaux, se la issât aller à chanter des victoires de village en village, ou de gracieuses scènes dramatiques, ou d'imposans tableaux de cette forte nature contre laquelle il eut souvent à lutter sans témoins et sans secours (...) Ma is là où l'écriture n'est pas chargée de la conserva tion des souvenirs, la mémoire des hommes est tenace et reconna issa nte, et il me sembla it tout à fa it impossible qu'un grand nombre de monumens littéraires anciens ne se fussent pas perpétués d'âge en âge, confiés à la garde des cœurs basques. Chant hérö̈que, scène domestique, la i d'a mour, je fera is appel à tout; tout me paraissa it bon à obtenir, pourvu qu'il portât un caractère d'originalité et non d'imitation. (J-A-C. Buchon, Représentation d'un mystère dans le Pays Basque, Le Mémorial des Pyrénées, 31 octobre et 2 novembre 1839, passage en gras souligné par BO)

Les références que Buchon évoque dans son texte, Robert Burns et James Hogg (le berger d'Ettrick), sont écossaises et d'origine paysanne (bien qu'il n'y ait pas dans leur cas de translation linguistique, car elles sont relativement récentes). Même si le modèle est celui du barde manifestant à travers chants et poèmes l'expression originale et authentique du peuple, Buchon ne semble pas enclin à la complaisance à l'égard des escroqueries littéraires que la quête de ce type de textes a pu susciter depuis le XVIIIe siècle. En effet, et le fait est probablement significatif s'agissant de références écossaises, Buchon évite de mentionner Macpherson, contrairement à ce qu'avait fait quatre ans plus tôt Garay de Monglave à propos du Chant d'Altabizkar. Si, donc, Buchon est prudent en cette matière, comme semble l'indiquer son attitude à propos des bardes écossais, faut-il alors admettre sa datation du manuscrit de Clovis ? Avant de répondre, considérons d'autres éléments liés à cette question.

32 - Pour une classification des différents types de falsifications dans les chants populaires en Europe, voir J.A Cid (1994). 
§8.3. Dans son article Buchon dresse la liste des auteurs de pastorales dont le souvenir a été conservé dans le pays, et il cite en premier lieu un certain Bordet d'Alos, qui viva it vers 1500.

Il en est de ces pastora les na tiona les comme des chants na tiona ux; les chants se conservent, et les noms des a uteurs s'oublient. Tout ce que j'ai pu savoir jusqu'ici, c'est qu'on a conservé le souvenir de: Bordet d'Allos (sic), qui viva it vers 1500 ; Etchegoyen de Laruns, qui viva it il y a une centaine d'années; Etchegoyen d'Ibarolle, Navarrais, mort il y a une cinquantaine d'années; Etchevès de Viodos, mort récemment; Louis-Pierre-Saffores vivant encore à Tardets (Représentation d'un mystère dans le Pays Basque, Mémorial des Pyrénées, 1831, n¹32, passage en gras souligné par BO)

L'information concernant Bordet est pour le moins étonnante et demande, elle aussi, à être examinée de plus près. En effet, il est rapporté que le souvenir du nom d'un auteur dramatique souletin vivant vers 1500 avait été conservé parmi ses compatriotes, ainsi que celui de son origine villageoise (Alos), durant près de trois siècles et demi, jusqu'au milieu du XIXe siècle, sans qu'aucune mention d'aucune sorte en soit connue par ailleurs. La chose soulève le scepticisme, notamment si pour admettre une telle hypothèse il faut se fonder sur un témoignage unique et isolé : non pas un témoignage qui aurait été laissé quelque auteur plus ancien, et donc plus proche des époques évoquées, très bon connaisseur de la Soule et de la littérature basque ancienne comme Oihenart, mais par un voyageur du XIXe siècle ayant passé moins d'une semaine dans le pays, dont il ignorait la langue et à peu près tout de sa littérature. Poser la question, c'est y répondre. Ceci paraît fort peu vraisemblable. ${ }^{33} \mathrm{Et}$ il convient alors de s'interroger : puisque la date de 1500 évoquée à propos de Bordet est exactement celle attribuée au manuscrit de Clovis, ne faut-il voir là qu'une simple coïncidence ? Pour le moins cela montre que les indications de Buchon en matière de datations relatives aux tragédies souletines ont été faites avec une certaine légèreté.

Autre élément à considérer, qui, me semble-t-il, confirme la nécessaire remise en question des dires de Buchon : le fait d'avoir trouvé ce manuscrit de l'an 1500 chez un simple instituteur de pastorales, Jean Pierre Saffores, dont l'état habituel, nous dit-il, est de fabriquer des souliers. Ce Saffores (qui est le même que le copiste du manuscrit de Sa int Ja cques analysée au \$7) apparaît, effectivement, dans la première moitié du XIXe siècle comme pastoralier, et il occupe une place importante dans la vie de ce théâtre durant quelques décennies (Oyharçabal 1983). Lorsqu'il le visita, Saffores possédait, nous dit Buchon, 70 manuscrits, dont il n'était pas l'auteur pour la plupart, même s'il en composa également. Il est déjà difficile d'expliquer un vide de deux siècles et demi dans les manuscrits de pastorales, mais si une telle rupture devait être admise, on conviendra que la conservation du manuscrit ancien unique devrait résulter d'une cause particulière, c'est-à-dire d'un mode de conservation qui l'aurait mis à l'abri des vicissitudes des autres copies de tragédies qui durant deux cent cinquante ans se seraient, elles, toutes perdues. Mais alors, si l'on se situe dans un tel contexte, comment ce manuscrit se serait-il retrouvé en possession d'un modeste cordonnier de Tardets, dont rien n'indique d'ailleurs qu'il appartenait à une lignée de pastoraliers ancienne. ${ }^{34}$

33 - Hérelle (1922, p. 347) avait bien sûr relevé le fait, mais estimait que l'erreur qu'il soupçonnait était due au fait que le témoignage des informateurs de Buchon (en fait Saffores lui-même certainement) n'était pas fiable : car, disait-il, les Basques s'embrouillent aisément dans leur millésimes. L'idée que de modestes artisans souletins puissent en plein milieu du XIXe siècle situer spontanément un auteur de tragédie populaire du passé en l'an 1500 laisse perplexe, d'où ma préférence pour l'hypothèse évoquée dans le texte, selon laquelle Buchon avait plutôt lu ce nom sur le manuscrit de Clovis.

34 - Buchon mentionne bien un Louis-Pierre Saffores dans la liste des pastoraliers dont on a conservé la mémoire, mais il vivait encore à Tardets, nous dit-il, lors de sa visite. 
Si l'on refuse de croire Buchon sur parole, tout en écartant l'hypothèse de la supercherie, il est possible que, s'agissant pour Buchon d'une question qu'il n'étudia qu'occasionnellement et certainement avec quelque légèreté à l'occasion d'une chronique de presse, l'indication ait résulté simplement d'une mauvaise lecture (facilitée par les croyances et les présupposés du temps en cette matière, que Buchon, comme nous l'avons vu, partageait), ou bien d'une appréciation erronée quant à la datation du document si aucune date n'y figurait. Je pencherais plutôt vers le premier membre de cette alternative. En effet, Bordet n'est pas un nom inconnu dans les études consacrées aux tragédies souletines, car il est mentionné en composition dans certains manuscrits de la fin du XVIII siècle : il apparaît comme le copiste qui, associé avec le régent Pierre Lahore de Barcus, avait endommagé le manuscrit d'Etchebarne (mss. 213 de la BNF) de Sa int Jean de Paris en 1777 (voir Hérelle 1923, p. 160, et Mozos 1994, p. 16, 27 et 464). Bordet était en fait le nom de la maison d'un certain Carriciry (lire Karrikiri) à Alos, d'où le nom composé apparaissant sur le manuscrit cité, suivant l'usage local associant le patronyme et le nom usuel correspondant lui-même au domonyme : Carriciry Bordet, comme pour le nom du chansonnier Topet Etchahoun.

Le scénario que l'on peut suggérer, en manière d'hypothèse, et avec toutes les réserves nécessaires s'agissant d'un document que nul n'a jamais vu en dehors de Buchon, ${ }^{35}$ est que le Bordet d'Alos apparaissant sur la copie du manuscrit de Clovis acheté par Buchon était celui apparaissant également dans la copie de Sa int Jean de Paris, de sorte que ce manuscrit de Clovis, malgré les indications de Buchon, devrait être également rapporté à la même époque.

\section{§9. Les surprenantes références aux acteurs navarrais de la fin du XVIe siècle.}

§9.1. En dehors des manuscrits, les témoignages de représentation peuvent constituer une autre source attestant de l'existence d'une tradition théâtrale populaire. Il en est ainsi par exemple des représentations de théâtre charivarique, qui sont connues par les descriptions qui en ont été faites ou les interdictions dont elles ont pu être l'objet, mais sans que cela ait donné lieu à la production de manuscrits comme cela a été le cas pour les tragédies ou même les pièces comiques correspondantes, appelées farces.

Les témoignages peuvent être de sources diverses. S'agissant de théâtre populaire, réalisé en marge des circuits de production culturelle classiques, on peut en distinguer deux sortes : les archives administratives ou ecclésiastiques, par exemple à travers les interdictions ou réprobations que les représentations pouvaient faire naître (comme par exemple en Gascogne ou en Bretagne) ${ }^{36}$, et les témoignages directs de ces représentations. Hélas, ces documents n'apparaissent que très tardivement dans le cas des tragédies souletines : bien plus tard que les premiers manuscrits. En effet, les premières interdictions attestées datent de la période révolutionnaire (Hérelle 1910) et les premiers témoignages directs de la fin du XVIIIe siècle, à travers trois brèves mentions apparaissant dans le manuscrit datant de 1785 d'un auteur souletin, J. Eguiateguy (Peillen 1981). Les premières

35 - Hérelle (1926a) indique avoir effectué diverses démarches, en particulier auprès des descendants de Buchon, pour essayer de retrouver ce manuscrit. Efforts demeurés, hélas, infructueux.

36 - En dehors du Pays Basque, voir par exemple le texte de l'arrêt de la cour de Rennes de 1753, publié par F.-M. Luzel dans son introduction à la publication de Sa inte Tryphine et le Roi Arthur (1863), et aussi, pour la Gascogne, Heiniger (1996) 
descriptions de spectateurs (d'ailleurs étrangers) sont encore plus tardives, puisqu'il s'agit de ceux de Buchon et Badé vers $1840 .^{37}$

Est-ce à dire qu'il n'y ait aucun document attestant de l'existence tragédies populaires basque avant 1750, date figurant sur le manuscrit de Sainte Elisabeth ? Encore une fois, la réponse dépend de l'interprétation de certains témoignages, et de leur évaluation.

La littérature à ce sujet a fait apparaître deux documents manuscrits dont on a pu penser, au moins dans un premier temps, qu'ils attestaient de l'existence des tragédies basques au XVIe siècle. Le premier que nous mentionnons fut publié par J.M. Floristán (1993) ; il s'agit d'une lettre adressée au représentant du Roi d'Espagne en Navarre, par Charles de Luxe en 1595. Le second fut publié par Lafitte (1967) : il s'agit d'un manuscrit datant de 1665 dans lequel Oihenart rédige le brouillon d'un art poétique basque, dans lequel il fait référence aux diverses traditions existant en matière de versification dans la littérature basque et où il mentionne explicitement l'existence d'un théâtre basque en Basse-Navarre au milieu du XVIe siècle.

Comme nous allons le voir chacun de ces deux documents pose des problèmes d'interprétation différents par rapport à la question d'une attestation d'un théâtre basque ayant pu servir de matrice aux tragédies populaires souletines.

§9.2. Il y a une dizaine d'années fut publiée par Floristán une série de lettres rédigées en basque rencontrées dans les archives espagnoles de Simancas. Il s'agissait de courriers d'information adressés secrètement depuis les provinces basques de France aux représentants du Roi d'Espagne en Navarre et en Gipuzkoa. L'une des ces lettres est écrite par Charles de Luxe (plus exactement par sa fille sous sa dictée $)^{38}$, et destinée selon toute vraisemblance au régent du Roi de Navarre faisant office de Vice-Roi, Calderón.

La lettre, non datée et fort brève, fut remise au représentant du Roi à Pampelune, par un certain Palacios qui l'avait reçue à Ochagavia. Elle était accompagnée d'une traduction espagnole. Le courrier original comporte un court passage que le traducteur de la lettre interpréta comme correspondant à une demande d'acteurs. Voici le passage concerné, accompagné de la traduction qui en fut faite à l'époque (Floristán, 1993, p. 204-5) :

suplicazen nizaucu auctore pareba (sic) na hi derautacun chercatu neure duhuruian. le suplico de un par de actores me quiera buscarlos en mi dinero

La traduction réalisée à la réception du message faisant allusion à une requête de deux acteurs, Floristán indique qu'il devait s'agir d'acteurs de tragédies populaires, comme elles sont connues en Soule :

37 - Humboldt également fit une référence aux tragédies traditionnelles dans ses notes écrites suite à son voyage de 1801. Toutefois il ne dit pas avoir assisté à une représentation, et paraît restituer des informations de seconde main, donnant même à entendre (du moins selon le texte publié dans la traduction espagnole) que les acteurs sont des deux sexes (Humboldt 1975, p.186). Cette dernière information, néanmoins, ne résulte probablement pas d'une erreur. En effet, il semble que durant cette période certaines tragédies aient été jouées par des acteurs des deux sexes. Comme A Loidi me le fait observer dans une communication personnelle, c'est ce qu'atteste le manuscrit de Clovis de 1799, dont Michel (1847) fournit la liste des acteurs ; voir aussi Hérelle 1910.

38 - Apparemment Charles de Luxe ne savait pas ou ne pouvait pas écrire. La lettre publiée est écrite par sa fille, selon ce qu'indique Palacios. Par ailleurs, ce dernier, dans un autre courrier postérieur daté de septembre, indiquera avoir reçu les informations oralement, car Charles n'a va it personne à la ma ison pour écrire (Floristán 1993). 
En fin, como detalle significativo de la conexión existente entre ambas vertientes pirenaicas cabe destacar la petición que se hace de dos actores, sin duda para las representaciones tea trales populares, género literario de gran arraigo en el Soule de la época y posterior. (Floristán, 1993, p. 182)

La mention d'acteurs dans cette missive constitue une information tout à fait inattendue. Dans une période particulièrement trouble et depuis une région soumise à des conflits politiques et religieux continuels depuis un siècle, Charles de Luxe prie le représentant de Philippe II en Navarre de lui envoyer une paire d'acteurs! La chose paraît même si naturelle, qu'aucune précision supplémentaire n'est ajoutée. Comment interpréter ce document ? Fait-il réellement allusion aux représentations dramatiques du temps, et par là peut-être aux tragédies telles qu'elles furent connues ensuite en Soule, comme le propose Floristán?

Ici encore, les apparences se révèlent trompeuses. Dans le texte original en basque, l'interprétation en cause repose sur un mot : auctore, que le traducteur contemporain, puis Floristán (1993) l'éditeur diplomatique, et enfin Satrústegui (1993) l'éditeur critique de la lettre, traduisent 'acteur'. Observons en premier lieu que le vocable n'appartient pas au fond lexical basque traditionnel recensé (il est absent du DGV) et qu'il s'agit d'un emprunt à une forme romane issue du lat. actore. ${ }^{39}$ Ceci est possible, mais il faut admettre une faute dans la graphie car une réalisation avec la diphtongue [aw] à l'initiale est problématique dans cet emprunt. Cette faute consisterait en une addition de lettre : a uctore pour actore, une erreur possible certes, mais guère facile. ${ }^{40}$ La leçon proposée est passablement coûteuse. Est-elle la seule possible?

Si l'on considère de plus près l'orthographe de la lettre de Charles de Luxe dans la transcription de Floristán, on remarque une chose : pour rendre l'occlusive vélaire sourde [k], seule la lettre $c$ est utilisée (jamais $k$, ou $q u$ ), alors que par ailleurs cette même lettre est employée pour rendre la sifflante dorsale [s] (dans la graphie de cette missive, la lettre $s$ rend la sifflante apicale comme aujourd'hui, et $z$ l'affriquée dorsale). On note par ailleurs l'absence totale de cédille sur la lettre $c$, quelle que soit sa valeur. Dans ce courrier, la lettre $c$ est donc utilisée de façon ambiguë pour restituer les sons [k] et [s] Je donne quelques exemples des deux correspondances (devant voyelle) :

- $c=[\mathrm{k}]$; adiscide 'ami', canpouan 'dehors', duce 'duc', principalci 'principalement'

- $c=[\mathrm{s}]$; cauri 'blessure' cegei 'à vous', coure 'votre', principalci 'principalement'

Ayant ceci à l'esprit, l'interprétation de auctore doit être revue, car ce mot (où $c$ est cette fois-ci devant consonne) peut se lire aussi désormais [awstore], ce qui nous éloigne de la signification proposée. En effet, auztore, qui est également un emprunt, mais sur l'occitan ancien austore, et qui appartient au lexique basque (sous plusieurs variantes ; voir l'entrée aztore dans le DGV), signifie 'autour' et est attestée sous cette forme chez Oihenart au XVIIe siècle. Le choix entre les deux interprétations ne laisse guère de place au doute : autant l'échange de comédiens, apparemment professionnels, des deux côtés d'une frontière conflictuelle comme la frontière navarraise de ce temps paraît improbable, autant la demande de deux oiseaux de chasse semble naturelle de la part du chef d'une des principales maisons de Basse-Navarre, que l'on imagine volontiers amateur de cette pratique de chasse.

39 - Dans les tragédies traditionnelles, le terme apparaît en tant qu'emprunt gascon ou français : aktür. 40 - On écartera une contamination à partir du lat. a uctore 'auteur' ici. 
La conclusion est sans ambiguité : la lettre de Charles de Luxe ne documente pas l'existence de pratiques théâtrales, d'aucune sorte.

\section{§10. Les attestations des pastorales basques du XVIe siècle en Basse-Navarre.}

$\S 10.1$. Le cas du second document pouvant attester de l'existence de pastorales au XVIe siècle est différent, car il est plus tardif et ne témoigne qu'indirectement du théâtre de ce temps. Il s'agit de l'Art poétique basque, manuscrit d'Oihenart daté de 1665 , où son auteur fait une mention explicite à une oeuvre appartenant à la poésie dramatique basque du XVIe siècle. Voici ce qu'indique Oihenart :

Il y Eust Un a utre prestre, na tif de St Jean Pied de port, nommé Mr Jean dEtchega ray qui s'adonna aussi a la poesie basque; Cest Lautheur de La pastorale Intitulée arza in gorria qui a été Iouee plusieurs fois en Cette Ville. Il escriuoit il y a Cent ans. Ia y Ueu Un Volume de ses rimes basques Escrit de sa main, La plus part de ses Uers sont a ussy composes de quinse syllabes, Et on les mesmes manquements, En la forme, que Ceux dEtchepare. (L'art poétique basque édité par Lafitte, 1967, p. 228)

Malgré l'éloignement (un siècle) de sa référence, et bien qu'aucun autre document ne nous soit parvenu attestant de l'existence de ce répertoire, le témoignage est difficilement contestable : Oihenart était un lettré et un érudit qui avait une excellente connaissance de la littérature basque, et une grande habitude de la lecture des archives et des manuscrits, et son indication, je crois, peut être considérée comme réellement fiable. An'en pas douter, cette citation nous assure de l'existence de pastorales en langue basque en Basse-Navarre au XVIe siècle. Ce point étant établi, il n'en résulte pas nécessairement que les tragédies souletines que nous connaissons en représentent une survivance, autrement dit, il nous faut nous interroger sur l'existence d'une continuation entre le théâtre du XVIe siècle dont parle Oihenart, et qu'il désigne sous le terme de pastorale, et les tragédies populaires souletines du XVIIIe siècle.

Lafitte (1974, p. 258) avait pris au pied de la lettre les dires d'Oihenart et considéré que ce dernier portait le premier témoignage concernant les pastorales (= tragédies) traditionnelles, ce qui renforçait l'idée, généralement admise par les auteurs depuis le XIXe siècle, que ce théâtre avait été connu hors de Soule également en des temps plus reculés et notamment au XVIe siècle.

Pourtant un examen attentif des indications fournies par Oihenart fait surgir le doute. Non pas quant à leur authenticité, mais quant à leur interprétation. Le mot clé est ici celui de pastorale dont nous avons dit qu'il était utilisé, à côté de celui de tragédie, dans la tradition souletine dès le XVIII siècle, et qu'il s'imposa ensuite progressivement au XIXe siècle dans le sens qu'il a encore aujourd'hui dans les études littéraires basques. Mais que désigne-t-il dans la citation d'Oihenart?

Pour Lafitte, ainsi que nous l'avons signalé, tout comme, avec des nuances significatives, pour Orpustan (1996, p. 131), le terme désigne le même genre dramatique qu'à l'époque moderne : le vocable se maintient et les formes théâtrales attestées en Soule à partir du XVIIIe siècle constituent une forme tardive d'un théâtre plus ancien ayant été pratiqué ailleurs, au moins en Basse-Navarre. Je crois plutôt pour ma part que cette interprétation doit être mise en doute car elle repose probablement sur un anachronisme lexical, favorisé par le fait qu'Oihenart était souletin, et les tragédies basques attestées, elles aussi, typiquement souletines et appelées également pastorales. 
§10.2. Tout ce que dit Oihenart au sujet de ce théâtre, concernant le lieu de représentation, le titre de la pastorale, l'auteur et la thématique contredit ce que l'on sait des tragédies traditionnelles souletines, de sorte que si chacun des arguments pris en particulier ne représente pas une objection insurmontable à l'hypothèse de la continuité ou de l'identité de la tradition dramatique, la conjonction de ces arguments la rend peu crédible. J'ai déjà eu l'occasion d'évoquer ces éléments, que je reprends ici : ${ }^{41}$

- Le lieu : les représentations mentionnées par Oihenart ont eu lieu dans une ville de Basse-Navarre, et, même s'il s'agit d'une petite ville, la cité de Saint-Jean-Pied-dePort pour ce temps évoque plus un théâtre de type urbain que rural. Au contraire, les tragédies traditionnelles anciennes correspondent typiquement à un théâtre rural.

- L'auteur : la pastorale de Saint-Jean-Pied-de-Port avait un auteur, dont le nom était encore connu d'Oihenart un siècle plus tard; à l'inverse la notion d'auteur est étrangère à la tradition populaire souletine ancienne, où les figures de régent, de propriétaire de copies, de copiste, et d'auteur ou adaptateur proprement dit sont indissociables, de sorte qu'un régent de tragédie a toute liberté de faire ce que bon lui semble avec les textes des copies qu'il possède. Par ailleurs, l'auteur est un prêtre, alors qu'aucune pièce du répertoire des tragédies traditionnelles n'a pour auteur un membre du clergé.

- Le titre: Oihenart cite une œuvre qui avait un véritable titre (Le berger rouge), formulé en basque, ne faisant référence à aucune histoire connue ; tout ceci est absolument étranger à la tradition des tragédies souletines, lesquelles n'ont pas de véritables titres, mais se désignent éventuellement dans les ex-libris, en français, par le nom du personnage principal (toujours connu par ailleurs) dont l'histoire ou la vie sont représentées, associé par le biais de la préposition de à un nom générique comme tragédie, pastorale, vie, ou histoire : tra gédie de sa inte Elisa beth, pa storale de Roland, vie de Charlemagne.

- La thématique : La pièce jouée à Saint-Jean-Pied-de-Port évoque une thématique pastorale au sens classique du terme puisque mentionnant dans son titre un berger. Or le répertoire des tragédies traditionnelles n'a rien à voir avec le genre pastoral en vogue au XVIe siècle, y compris dans le registre dramatique (notamment, mais pas uniquement, à travers les bergeries de Marguerite de Navarre).

Nous pouvons voir que rien dans les indications d'Oihenart ne vient établir quelque parenté que ce soit avec les tragédies traditionnelles, en dehors de la désignation (pastorale). Par ailleurs, eu égard, d'une part, au titre de la pièce, laquelle fait référence à un berger, d'autre part, au fait que cette pastorale appartenait selon toute vraisemblance à un répertoire d'auteur lettré (un poète qu'Oihenart juge utile de citer, fût-ce pour le critiquer, au même titre qu'Echepare, Haramburu ou Etcheberri de Ciboure), il est fort probable que le terme pastorale dans cette citation a pour contenu celui qu'il avait en français classique. La première édition (1694) du dictionnaire de l'Académie française en donne une définition on ne peut plus claire : pièce de théâtre dont les personnages sont des bergers et des

41 - Dans mes travaux antérieurs, j'ai évoqué l'hypothèse que ce que disait Oihenart concernant la versification d'Etchegaray pouvait se rapporter à son théâtre (Oyharçabal 1991, p. 8). Mais, après relecture, il m'apparaît que l'interprétation la plus naturelle de ces propos favorise plutôt une lecture où le volume de rimes basques de cet auteur constituait une production distincte de la pastorale elle-même. En ce cas, le fait que la critique des vers d'Etchegaray par Oihenart impliquât que ceux-ci étaient d'une forme très différente des versets de tragédie n'est pas significatif pour l'argumentation développée dans le texte. 
bergères. Selon les éléments dont nous disposons, il est vrai très peu nombreux, on peut donc supposer que la pastorale Le berger rouge d'Etchegaray appartenait à ce qui constituait l'un des trois genres majeurs de la comédie à cette époque, à savoir la tragédie, la tragicomédie et, donc, la pastorale. ${ }^{42}$ On le sait, ce terme, tout comme celui de tragédie avec lequel il fut longtemps en concurrence, fut utilisé par la suite comme terme générique pour désigner les représentations de théâtre populaire données en Soule et dans la région, mais il s'agit là d'un emploi dérivé, que l'on ne saurait attribuer également à Oihenart sans risquer de commettre un regrettable anachronisme lexical.

Aussi bien si le témoignage d'Oihenart permet de poser l'existence d'un théâtre en langue basque au XVIe siècle, ${ }^{43}$ il ne s'ensuit pas que les tragédies traditionnelles souletines en furent une continuation. Précisément, outre l'absence de documentation sur une période de deux siècles, un argument qui vient à l'esprit, allant dans le sens d'une absence de filiation, et par conséquent d'une introduction plutôt tardive de ce théâtre en Soule (dans la première partie du XVIIIe siècle ou à la fin du siècle précédent), est fourni précisément par ce manuscrit Oihenart datant, rappelons-le, de 1665. En effet, compte tenu de la qualité de l'auteur et de son intérêt pour la littérature, à la fois savante et populaire (chansons, proverbes et dictons) en langue basque, on imagine mal qu'il aurait tu, s'il l'avait simplement connue, l'existence de ce théâtre dans la région d'où il était lui même originaire et où il vécut. Les arguments reposant sur le silence des textes sont toujours faibles, mais il paraît peu vraisemblable que connaissant l'existence de tragédies et d'une tradition dramatique semblables à ceux attestés en Soule au siècle suivant, Oihenart, natif de cette province, et dont le propos dans son étude est de critiquer les usages littéraires de ses contemporains comme de ses prédécesseurs, les aurait tout simplement ignorés.

\section{§11. Conclusion.}

Cette recherche a permis d'établir que la tragédie Sainte Elisabeth de Portugal, que nous avons brièvement présentée à partir du manuscrit de 1750 est bien la pièce du répertoire des tragédies populaires traditionnelles la plus ancienne qui soit parvenue jusqu'à nous. Elle offre le plus ancien manuscrit, non seulement parmi ceux qui ont été conservés, mais également parmi ceux qui sont connus. En effet, les autres mentions de pièces prétendument plus anciennes correspondent à des erreurs (Saint Jacques, Jeanne d'Arc). En ce qui concerne la pastorale jouée à Saint-Jean-Pied-de-Port vers le milieu du XVIe siècle, nous pensons qu'elle ne représente pas une réelle objection à l'analyse présentée, car elle constituait selon toute vraisemblance une vraie pastorale, au sens classique de ce terme en français, et qu'elle appartenait à un autre genre théâtral que les tragédies traditionnelles souletines.

La question se pose de déterminer l'origine de cette tradition des tragédies populaires souletines : soit dans un ancien théâtre en langue basque qui aurait

42 - La première édition du dictionnaire de l'Académie française, à l'entrée comédie, apporte l'indication suivante : il [le mot comédie] se prend plus généralement pour toutes sortes de pièces de théâtre, comme sont la Tra gédie, la Tra gicomédie et la Pastorale.

43 - Il serait, je pense, excessif de restreindre l'existence d'un théâtre en langue basque au XVIe siècle au seul genre pastoral, à l'exclusion de tout autre, même si seul ce dernier est attesté. L'hypothèse contraire me paraît plus vraisemblable, même si bien sûr l'absence de documentation allant en ce sens nous empêche d'aller au-delà de la simple présomption. 
disparu plus précocement dans les autres provinces mais se serait maintenu en Soule, soit dans l'adaptation en Soule du théâtre populaire des campagnes qui vécut assez tardivement dans certaines régions, y compris en Gascogne, ${ }^{44}$ cette seconde hypothèse étant incontournable, si l'on admet, comme nous le faisons ici, une adoption plutôt tardive de cette tradition théâtrale par les Souletins. Il est évidemment difficile de prouver une absence de continuation entre le théâtre basque ayant existé de façon très probable au XVIe siècle au moins en Basse-Navarre et les tragédies traditionnelles qui ne nous sont connues que depuis le milieu du XVIII e siècle, mais dont nous avons indiqué qu'elles étaient certainement établies en tant que tradition avant 1750 . Bien que cela soit paradoxal, compte tenu du fait qu'il fut le premier à parler de pastorale dans le contexte du répertoire dramatique en langue basque, l'absence de référence spécifique chez Oihenart à un genre dramatique semblable aux tragédies traditionnelles souletines nous fait penser qu'au milieu du XVIIe siècle cette tradition n'était pas encore établie.

Je souhaiterais terminer cette recherche par quelques observations relatives aux textes littéraires basques qui, bien que n'appartenant pas au domaine de la littérature orale, ne sauraient se rattacher au monde de l'imprimé. Les tragédies populaires souletines en forment un exemple tardif très illustratif, mais il y eut certainement des antécédents, hélas moins bien attestés, dans des contextes différents. On sait que les productions littéraires en langue basque bas-médiévales ou postérieures reposant sur la seule oralité ne sont parvenues jusqu'à nous que de façon très limitée et souvent fragmentaire (Michelena 1960), ce qui se comprend sans difficulté. Mais, même en dehors de ce cas de figure, l'idée d'une rupture dans la transmission de certaines pratiques littéraires post-médiévales en langue basque impliquant un support papier ne devrait pas surprendre, bien que très peu de traces aient été conservées de ces écrits produits en dehors des circuits liés à l'impression, sur lesquels pesaient des contraintes matérielles et sociales spécifiques. Tout récemment, on a découvert un manuscrit, rédigé en langue basque dans sa plus grande partie, datant probablement des années 1565 et contenant diverses poésies et une partie d'un roman pastoral. L'auteur, ou à tout le moins le copiste, dénommé Perez de Lazarraga, était un membre d'une grande famille originaire du nord de l'Alava, et certains ce sont beaucoup étonnés de l'existence d'un tel document, considérant comme une surprise qu'il pût y avoir un tel usage littéraire du basque au XVIe siècle dans cette région. Mais l'extrême rareté de tels documents manuscrits ne doit pas donner lieu à des interprétations inappropriées. Il est en effet difficile d'imaginer que dans les usages autres que ceux réservés aux langues de l'imprimé, dont le basque pour des raisons de statut ne faisait pas partie à cette époque, sinon marginalement dans les provinces nordorientales, il n'y eut pas d'emploi de la principale langue véhiculaire du pays ( $\mathrm{du}$ moins sur une partie importante de son territoire), dans des emplois esthétiques et récréatifs, plus ou moins socialisés. Même si, évidemment, la maîtrise de l'écrit n'était pas - beaucoup s'en fallait - généralisée à l'ensemble de la population, on a peine à croire que dans une société où cet outil était nécessairement bien présent, il aurait été exclu de ces emplois proprement littéraires parmi les couches sociales pouvant en disposer. La pastorale d'Etchegaray, qui fut jouée plusieurs fois à Saint-

44 - Heiniger (1999) fait le point sur cette question, soulignant l'existence d'une tradition théâtrale populaire, principalement francophone à cette époque, à côté d'un théâtre d'auteur en langue occitane. L'hypothèse d'une introduction de ce théâtre en Soule depuis la Gascogne n'implique donc pas que cette source première fut un théâtre linguistiquement occitan. Des difficultés demeurent cependant, liées notamment au mode de composition des tragédies souletines, et qui demandent à être éclaircies. 
Jean-Pied-de-Port au XVIe siècle montre par exemple l'existence en Basse-Navarre d'une vie théâtrale certainement indépendante de l'écrit imprimé, et il est probable qu'il en fut de même dans le registre poétique en Basse-Navarre et en Soule (selon diverses indications d'Oihenart), mais certainement ailleurs également, bien que les preuves directes soit moins fournies qu'on ne le souhaiterait : poèmes navarrais et biscayens, et désormais, donc, le manuscrit de Perez de Lazarraga. Les études littéraires basques ont eu souvent tendance à considérer comme appartenant à la pure oralité tout ce qui ne relevait pas de l'imprimé, mais cette démarche, que justifie une absence de frontière nette en bien des cas entre ces deux domaines, peut se révéler trop réductrice. Précisément, les tragédies souletines témoignent, dans le registre de la littérature populaire, des limites de ce type d'opposition : car évidemment les tragédies traditionnelles, que l'on a parfois classées dans les études basques comme relevant de la littérature orale (Lekuona 1935), mais qui pouvaient comporter comme Sainte Elisabeth près de 1600 quatrains, bien qu'absolument étrangères au monde de l'imprimé, n'existèrent et ne se transmirent que grâce au support de l'écrit.•

\section{Bibliografia}

- Agirre, P. 1996. Athanase Bela peire : Ca techima laburra (1696) : Autorearen garaia, norta suna eta idazla nak, grafiak eta fonologia. Edizio kritikoa eta hiztegia, Thèse, Universitad del País Vasco, Vitoria-Gasteiz.

- Alford, V. 1951. 'Une pastorale basque en 1951', Eusko-Jakintza, 3-6, MayatzAbenduak, Bayonne, 158-164.

- Badé, J. 1841, 'Un échantillon du théâtre populaire des Basques', Observateur des Pyrénées, octobre, $\mathrm{n}^{\circ} 745,747,748,751,753,754$, Pau.

- Bidart, P. 2001. La singularité basque, Presses Universitaires de France.

- Bilbao Telletxea, G. 1996. 'Jean Mekol Garindañekoaren Edipa pastorala', ASJU, XXX-1, 239-332.

- Bladé, J-F. 1866. Dissertation sur les chants hérö̈ques des Basques, A Franck, Paris.

- Bladé, J-F. 1869. Etudes sur l'origine des Ba sques, Paris, reprint Slatkine-Megariotis, Genève, 1976.

- Buchon, J.-A-C. 1839. 'Représentation d'un mystère dans le Pays basque', Mémorial des Pyrénées, ${ }^{\circ} 131-132,31$ octobre et 2 novembre, Pau.

- Chaho, A. 1856. Biarritz entre les Pyrénées et l'Océan, 2 tomes, édition en facsimilé 2004, Le livre d'Histoire, Paris

- Cid, J. A 1994. 'Tradición apócrifa y tradición hipercrítica en la balada tradicional vasca. I. Las falsificaciones de cantos populares en Europa ; ensayo de tipología', ASJU, XXVIII-2, 505-524.

- DGV. 1987-2004. Diccionario General Vasco, 15 tomes parus, Euskaltzaindia, Bilbao.

- Dubarat, V. 1907. 'Lettres adressées au capitaine Duvoisin', RIEB, 49-55.

- Duvoisin, J. 1841a. 'La poésie dramatique des Basques', Album Pyrénéen, mars, Pau, 90-102.

- Duvoisin, J. 1841a. 'Comédie des Basques', Album Pyrénéen, Pau, 207-215.

- Eguiateguy, J. 1785 [1983]. Lehen liburia edo filo so fo huskalduna ren ekheia, édition de T. Peillen, Euskaltzaindia, Bilbao.

- Floristán Imízcos, J. M. 1993. ‘Conflictos fronterizos, espionaje y vascuence a finales del siglo XVI : 20 documentos inéditos', Fontes Linguae Va sconum, 63, 177-219.

- Gavel, H. 1911. 'A propos du chant du prologue dans les pastorales', Revue Internationale des Etudes Basques, 533-537. 
- Haritschelhar, J. 1969. Le poète souletin Pierre Topet-Etchahun (1786-1862), Bayonne.

- Heiniger, P. 1999. 'La pastorale dans le jeu des langues', Théâtre populaire européen, cahier Oihenart 16, Eusko Ikaskuntza, Donostia - San Sebastian, 51-61.

- Hérelle, G. 1910. 'Les représentations de pastorales basques dans la Soule pendant la période révolutionnaire', RIEB, IV, 05-17.

- Hérelle, G. 1922. La représentation des pastorales à sujet tragique, Bulletin de la Société des Sciences Lettres et Arts de Bayonne, Bayonne, Bulletin de la Société des Sciences, Lettres et Arts de Bayonne, 189-362, publié en 1923 sous le même titre chez Champion, Paris.

- Hérelle, G. 1926a. 'Une pastorale de l'an 1500', Revue Internationale des Etudes basques, XVII-1, Eusko Ikaskuntza, Donostia - San Sebastian.

- Hérelle, G. 1926b. Les pastorales à sujet tragiques considérées littérairement, Champion, Paris.

- Hérelle, G. 1928. Le répertoire du théâtre tragique, Ca ta logue a na lytique, Société des Sciences, Lettres, Arts \& Etudes Régionales de Bayonne, Bayonne.

- Humboldt, W. von. 1875. Los va scos : a puntaciones sobre un viaje por el Pa ís Vasco en primavera del a ño 1801, traduction de T. de Aranzadi, Auñamendi, DonostiaSan Sebastián.

- Juaristi, J. 1987. El linea je de Aïtor, Taurus, Madrid.

- Lakarra J., Biguri K. \& Urgell B. 1983 Euskal baladak, 2 tomes, Hordago, Donostia-San Sebastián.

- Lafitte, P. 1967. L'art poétique basque d'Arnaud d'Oyhénart (1665), Gure Herria tiré à part, Bayonne, 195-234.

- Lafitte, P. 1974. 'Herri-literaturaz', chapitre d'un ouvrage collectif intitulé Lur eta Gizon, Euskal Herria, Jakin, Oñate (Gipuzkoa), 243-264.

- Lebraz, A 1905. Le théâtre celtique, sans éditeur.

- LeDuc, G. 1999. 'Les aspects populaires du théâtre populaire breton', Théâtre populaire européen, cahier Oihenart 16, Eusko Ikaskuntza, Donostia - San Sebastián, 17-49.

- Lekuona, M. 1935. Literatura oral vasca, 3ème édition, Kardaberaz, 22-B, sans date, Tolosa (Gipuzkoa).

- Léon, A 1909. Une pastorale basque, Hélène de Constantinople, Etude historique et critique.

- Loidi, A 1996. 'Santa Catherina pastoralaren XIX. mendeko bi eskuizkiribu', ASJU, XXX-2, 497-603.

- Luzel, F.-M. 1863. Sa inte Tryphine et le Roi Arthur, mystère breton en deux journées et huit actes, traduit, publié et précédé d'une introduction de F.-M. Luzel, T . Clairet, Quimperlé ; édition fac-similée de 2002, Presses Universitaires de Rennes, Terre de brume, Rennes.

- Luzel, F.M. 1994. Journal de route et lettres de mission, publié par F. Morvan, Presses Universitaires de Rennes, Terre de brume, Rennes.

- Michel, (dit Francisque-Michel). 1847. Introduction précédant l'édition des Proverbes basques recueillis par Arnauld Oihenart suivis des poésies basques du même a uteur, Jannet et Franck, Paris.

- Michel, (dit Francisque-Michel). 1857. Le Pays Basque, sa population, sa langue, ses mœ urs, seconde édition 1981, Elkar, Bayonne.

- Michel, (dit Francisque-Michel). 1854. "Lettre à M.P. Mérimée sur les représentations dramatiques en Pays Basque", Le Messager de Bayonne, 14 décembre.

- Michelena, L. 1960. Historia de la litera tura vasca, Minotauro, Madrid. 
- Mozos Mujika, I. 1994. Jean de Paris pastorala. Eskuizkribuen azterketa eta iturriaren moldamoduak, Thèse de philologie basque, Université du Pays Basque, Vitoria-Gasteiz.

- Mujika, J.A 1997. Euska lortografia ren hasta penak Iparraldeko litera turan, thèse de philologie basque, Universidad del País Vasco, Vitoria-Gasteiz.

- Oihenart, A 1665. L'Art poétique basque, manuscrit édité par P. Lafitte, Edition 'Gure herria', 1967.

- Orpustan, J-B. 1996. Précis d'histoire littéraire basque, Izpegi, Saint-Etienne-deBaïgorry.

- Oyharçabal, B. 1991 [1981]. La pastorale souletine : édition critique de Charlemagne, publication de thèse (sans la traduction du texte), 1981, 3 tomes, Université de Bordeaux 3, suppléments de ASJU, XVI, 1991.

- Oyharçabal, B. 1983. 'Deux pastoraliers du XIXe siècle', Bulletin du Musée Basque, Bayonne, 109-118.

- Oyharçabal, B. 1985. Zuberoako Herri Teatroa, Antropologia euskal bilduma, Haranburu, Donostia - San Sebastián.

- Oyharçabal, B. 1999. 'Noizkoak diren Zuberoako pastoralei dagozkien lehen dokumentuak ?', V. Herri-Literatura Jardunaldiak, Herri antzerkiaz, Euskera, Euskaltzaindia, Bilbao, 69-88.

- Peillen, T. 1981. 'Euskal antzerki zaharrenak', Euskera, Euskaltzaindia, Bilbao, 699-706.

- Quellien, N. 1889. Chansons et danses des Bretons, Maisonneuve \& Leclerc, Paris.

- Satrústegui, J. M. 1993. Relectura de los textos vascos de espionaje del siglo XVI, FLV, 443-475.

- Saroïhandy, J.D. 1927. La pa storale de Roland, separata BSSLAB, Bayonne.

- Segurola, K. 2002. 'Jeanne d'Arc pastoralaren eskuizkribua : le plus ancien connu?', Lapurdum, VII, 313-326.

- Tartas, I. 1666. Onsa Hilceco Bidia, édition de P. Altuna, 1995, Université de Deusto, Bilbao.

- Tartas, I. 1672. Arima penitentaren occupatione devotac, édition de P. Altuna, 1996, Mensajero, Bilbao.

- Urkizu, P. 1990. 'Jundane Jakoberen trageria', Bilduma, Revista del Servicio de Archivo del Ayuntamiento de Errenteria, Txertoa, San Sebastián, 10, 187-294.

- Urkizu, P. 1994. 'Jundane Jakobe pastoralaren maileguak', in J.-B. Orpustan (éd.) La la ngue basque parmi les a utres, Izpegi, Saint-Etienne-de-Baïgorry, 25-42.

- Urkizu, P. 1996. Historia del tea tro va sco, Euskal gaiak, La Navarra.

- Urkizu P. (dir.), Olaziregi M.J., Altzibar X., Aldekoa I., Garcia, J.R. Chueca J. 2000. Historia de la litera tura va sca, UNED, Madrid.

- Vinson, J. 1883. Folklore du Pays Basque, Paris.

- Vinson, J. 1884. Notice bibliographique sur le folklore du Pays Basque, Maisonneuve, Paris.

- Vinson, J. 1891. Saint Julien d'Antioche, pastorale basque, Bordeaux.

- Webster, W. 1899. 'Les pastorales basques', ouvrage collectif sans éditeur, La tradition a u Pays Basque, seconde édition 1994, 243-261, Elkar, Bayonne. 